\title{
Skew Brownian Diffusions Across Koch Interfaces
}

\author{
Raffaela Capitanelli ${ }^{1} \cdot$ Mirko D'Ovidio $^{1}$
}

Received: 19 January 2016 / Accepted: 24 August 2016

(C) Springer Science+Business Media Dordrecht 2016

\begin{abstract}
We consider planar skew Brownian motion (BM) across pre-fractal Koch interfaces $\partial \Omega^{n}$ and moving on $\overline{\Omega^{n}} \cup \Sigma^{n}=\Omega_{\varepsilon}^{n}$ where $\Sigma^{n}$ is a suitable neighbourhood of $\partial \Omega^{n}$. We study the asymptotic behaviour of the corresponding multiplicative functionals when thickness of $\Sigma^{n}$ and skewness coefficients vanish with different rates. Thus, we provide a probabilistic framework for studying diffusions across semi-permeable pre-fractal (and fractal) layers and the asymptotic analysis concerning the insulating fractal layer case.
\end{abstract}

Keywords Brownian motion · Additive functionals · Boundary value problems · Fractals

Mathematics Subject Classifications (2010) $60 \mathrm{~J} 65 \cdot 60 \mathrm{~J} 55 \cdot 35 \mathrm{~J} 25 \cdot 28 \mathrm{~A} 80$

\section{Introduction}

State of the Art Diffusions on irregular domains have been investigated by many authors as well as the construction of reflecting Brownian motions on non smooth domains $([9,22$, 29, 30]). However, if the domain $D$ is Lipschitz, then we can construct the usual reflecting $\mathrm{BM}$ as in [9]. Let $D \subset \mathbb{R}^{d}, d \geq 2$, a bounded Lipschitz domain. Existence and uniqueness of the solution to $d X_{t}=d B_{t}+\mathbf{n}\left(X_{t}\right) d L_{t}^{\partial D}$ have been investigated in [6, 7] when $\mathbf{n}(z)$ is the inward normal vector at $z \in \partial D$ and $L_{t}^{\partial D}$ is the local time of $X$ on the boundary of $D$. In particular, $L_{t}^{\partial D}$ is a non-decreasing process such that $\int_{0}^{\infty} \mathbf{1}_{D}\left(X_{t}\right) d L_{t}^{\partial D}=0$ that

P.U. Sapienza Università di Roma 2015. The authors are members of GNAMPA (INdAM).

Mirko D’Ovidio

mirko.dovidio@uniroma1.it

Raffaela Capitanelli

raffaela.capitanelli@uniroma1.it

1 Dipartimento di Scienze di Base e Applicate per l'Ingegneria, "Sapienza" Università di Roma, Via A. Scarpa 16, 00161 Roma, Italy 
is, the process does not increase inside $D$. The local time can be associated with the surface measure $([8,9])$ in the sense of the Revuz correspondence. Moreover, convergence of reflecting BM in varying domain has been also investigated (see for example [14] and the references therein). In [8] the authors studied the Robin problem on fractal domains in the framework of the so called trap domains (see [15]) which is a nice property to deal with for our purposes. We also deal with processes which are skew diffusions. The skew BM has been introduced in $[33,34,54]$ and constructed to model permeable barrier in [44, 45]. An interesting surveys can be found in [39]. It has been also investigated by many researchers as a tool in applied sciences. Applications to a single interface have been developed in [4, $33,34,40,43,46,54]$. Recent results on multidimensional skew BM can be found in [5, $52,53]$. In $[38,55]$ the authors approach homogenization problems. As well described in [34, pag. 272], it is possible to construct a reflecting $\mathrm{BM} B^{+}$on $\Omega \subset \mathbb{R}^{2}$, by considering a BM $B$ on $\mathbb{R}^{2}$ and the occupation time $\mathfrak{f}$ of $B$ on $\Omega$. That is, $B\left(\mathfrak{f}^{-1}\right)$ is identical in law to $B^{+}$. It is also shown in [34] that by killing $B\left(\mathfrak{f}^{-1}\right)$ at a random time $T$ with conditional law $\mathbb{P}\left(T>t \mid B\left(\mathfrak{f}^{-1}\right)\right)=\exp -\int \ell\left(\mathfrak{f}^{-1}(t), x\right) \kappa(d x)$, one obtains the connection with the motion driven by the Feynman-Kac generator ( $\ell$ is a local time and $\kappa$ is a killing rate). An interesting connection has been also given by verifying a conjecture of Feller. Indeed, an elastic BM on $[0, \infty)$ with elastic condition $\gamma u(0)=(1-\gamma) u^{\prime}(0), \gamma \in(0,1)$ is identical in law to $B^{+}$ killed according with the conditional law $\mathbb{P}\left(T>t \mid B^{+}\right)=\exp -\frac{\gamma}{1-\gamma} \ell^{+}(t, 0)$. We notice that the special cases $\gamma=1$ or $\gamma=0$ correspond to Dirichlet or Neumann conditions.

Our Results In this paper we consider boundary value problems on snowflake domain $\Omega$ by using the homogenization results obtained in $[19,20]$ with the approach of insulating layers (see, for example, [1, 13] in smooth layers). More precisely, the fractal layer is approximated by a two-dimensional insulating thin layer $\Sigma^{n}$ with vanishing thickness and decreasing conductivity. Therefore, the emerging operators have discontinuous coefficients on the pre-fractal interfaces $\partial \Omega^{n}$ and so we consider skew Brownian motions, that is generalized diffusions processes (see, for example, [44, 45] and [53]).

More precisely, the process we are dealing with is a skew planar BM on a bounded domain $\Omega_{\varepsilon}^{n}=\overline{\Omega^{n}} \cup \Sigma^{n}$ with pre-fractal interface $\partial \Omega^{n}$. We say that the BM in $\Omega_{\varepsilon}^{n}$ is skew meaning that it has different probability to stay in either $\overline{\Omega^{n}}$ or $\Omega_{\varepsilon}^{n} \backslash \Omega^{n}$. We have a skewness condition on the boundary $\partial \Omega^{n}$. We denote by $B_{t}^{\nu, *}$ the skew (modified) planar BM on $\Omega_{\varepsilon}^{n}$ and we focus on the multiplicative functional $M_{t}^{n}=\mathbf{1}_{\left(t<\zeta \Omega_{\varepsilon}^{n}\right)}$ of $B_{t}^{\nu, *}$ where $\zeta^{\Omega_{\varepsilon}^{n}}$ is the lifetime of $B^{\nu, *}$ on $\Omega_{\varepsilon}^{n}$ and $v$ is the skewness parameter (see Section 5).

In our analysis, we mainly focus on occupation measures and stopping times. A key role is played by the fact that the pre-fractal and fractal Koch domains are non trap. Thus, the fact that the semi-permeable barrier is given by the pre-fractal curve $\partial \Omega^{n}$ does not affect our discussion in terms of occupation measures. Let $T$ be the lifetime of the skew Brownian motion $B^{v, *}$ and $c_{n}$ be a sequence of positive constants describing the transmission condition on $\partial \Omega^{n}$. Under the non-restrictive assumption that $T=T_{c_{n}}$ (that is the lifetime depends on $c_{n}$ ) we consider the lifetime $\widehat{T_{c_{n}}}$ with conditional law $\mathbb{P}\left(\widehat{T_{c_{n}}}>t \mid B^{\nu, *}\right)=$ $\exp -c_{n} \sigma_{n} \int_{\partial \Omega^{n}} \ell_{t}^{n}(y) d \mathfrak{s}$ (see Section 5.1) where $\sigma_{n}$ is a structural constant associated with the arc-length measure $\mathfrak{s}$ on the pre-fractal boundary. In particular, we consider a sequence of exponential random variables $\zeta^{n}$ with parameter $c_{n} \in[0, \infty]$ from which we construct a sequence of stopping times $\widehat{\zeta^{n}}$ (see formula (6.1) below) depending on the time the process spends on (or cross) the pre-fractal interfaces.

Our aim is to investigate the asymptotic behaviour of $M_{t}^{n}$ when thickness (of $\Sigma^{n}$ ) and skewness coefficients vanish with different rates according with $c_{n}$. We show that the limit 
process can be the elastic, reflecting or absorbing Brownian motion according to the asymptotic behaviour of the parameter $c_{n}$ (see Theorem 10). Our approach is based on the study of the asymptotic behaviour of $\mathbf{1}_{\left(t<\zeta \Omega^{n}\right)}$ or equivalently $\widehat{M_{t}^{n}}=\mathbf{1}_{\left(t<\widehat{T_{c_{n}}}\right)}$.

Concerning the Dirichlet problem on $D \subset \mathbb{R}^{d}$, the connection between variational and probabilistic approach to diffusion equations with killing has been investigated for example in [10]. Boundary value problems with varying domains has been also investigated in [16, 50] where a key role is played by the capacity induced by a regular Dirichlet form.

Plan of the Work The plan of the paper is the following. Section 2 introduces notation and definitions of the pre-fractal and fractal Koch curves. Moreover, we recall the homogenization results obtained in [20]. Section 3 gives some basic aspects about positive continuous additive functionals and random times. In Section 4 we consider skew BM across a regular layer. The skew BM across irregular boundaries is introduced in Section 5. Our main results are collected and discussed in Section 6.

\section{Notation and Preliminary Results}

In this section we introduce the notation and some preliminary results. We recall the definition of the Koch curve with endpoints $A=(0,0)$, and $B=(1,0)$. We consider the family $\Psi^{\alpha}=\left\{\psi_{1}^{\alpha}, \ldots, \psi_{4}^{\alpha}\right\}$ of contractive similitudes $\psi_{i}^{\alpha}: \mathbb{C} \rightarrow \mathbb{C}, i=1, \ldots, 4$, with contraction factor $\alpha^{-1}, 2<\alpha<4$,

$$
\begin{array}{ll}
\psi_{1}^{\alpha}(z)=\frac{z}{\alpha}, & \psi_{2}^{\alpha}(z)=\frac{z}{\alpha} e^{i \theta(\alpha)}+\frac{1}{\alpha}, \\
\psi_{3}^{\alpha}(z)=\frac{z}{\alpha} e^{-i \theta(\alpha)}+\frac{1}{2}+i \sqrt{\frac{1}{\alpha}-\frac{1}{4}}, & \psi_{4}^{\alpha}(z)=\frac{z-1}{\alpha}+1,
\end{array}
$$

where $\theta(\alpha)=\arcsin \left(\frac{\sqrt{\alpha(4-\alpha)}}{2}\right)$.

By the general theory of self-similar fractals (see [27]), there exists a unique closed bounded set $K_{\alpha}$ which is invariant with respect to $\Psi^{\alpha}$, that is,

$$
K_{\alpha}=\cup_{i=1}^{4} \psi_{i}^{\alpha}\left(K_{\alpha}\right) \text {. }
$$

We recall that $K_{\alpha}$ supports a unique self-similar Borel measure

$$
\mu_{\alpha} \text { which is equivalent to the } d_{f}-\text { dimensional Hausdorff measure }
$$

where $d_{f}=\frac{\log 4}{\log \alpha}$. Let $K^{0}$ be the line segment of unit length that has as endpoints $A=(0,0)$ and $B=(1,0)$. We set, for each $n$ in $\mathbb{N}$,

$$
K_{\alpha}^{1}=\bigcup_{i=1}^{4} \psi_{i}^{\alpha}\left(K^{0}\right), \quad K_{\alpha}^{2}=\bigcup_{i=1}^{4} \psi_{i}^{\alpha}\left(K_{\alpha}^{1}\right), \quad \ldots, \quad K_{\alpha}^{n+1}=\bigcup_{i=1}^{4} \psi_{i}^{\alpha}\left(K_{\alpha}^{n}\right)
$$

$K_{\alpha}^{n}$ is the so-called $n$-th pre-fractal curve. Moreover, the iterates $K_{\alpha}^{n}$ converge to the selfsimilar set $K_{\alpha}$ in the Hausdorff metric, when $n$ tends to infinity. Let $\Omega^{0}$ be the triangle with vertices $A=(0,0), B=(1,0)$, and $C=\left(\frac{1}{2},-\frac{\sqrt{3}}{2}\right)$. We construct on the side with endpoints $A$ and $B$ the pre-fractal Koch curve defined before, which will be denoted by $K_{1, \alpha}^{n}$ and the Koch curve defined before, which will be denoted by $K_{1, \alpha}$. In a similar way, we construct on the other sides the analogous pre-fractal Koch curves (the Koch curves) denoting by $K_{2, \alpha}^{n}$ and $K_{3, \alpha}^{n}$ (by $K_{2, \alpha}$ and $K_{3, \alpha}$ ) the curves with endpoints $B$ and $C$, and $C$ 
and $A$, respectively. We denote by $\Omega_{\alpha}^{n}$ the pre-fractal domain that is the set bounded by the pre-fractal Koch curves $K_{j, \alpha}^{n}, j=1,2,3$. Moreover, we denote by $\Omega_{\alpha}$ the snowflake that is the set bounded by the Koch curves $K_{j, \alpha}, j=1,2,3$ (see Fig. 1). We denote by $\Sigma_{1}^{0}$ the open set condition triangle of vertices $A=(0,0), B=(1,0)$ and $C=(1 / 2, b / 2)$ where $b=\tan \left(\frac{\theta}{2}\right)$.

Following the construction in [18], for every $n$ and $\varepsilon$, we define the fiber $\Sigma_{1, \alpha}^{n}$, $\varepsilon$-neighborhood of $K_{1, \alpha}^{n}$ to be the (open) set

$$
\Sigma_{1, \alpha}^{n}=\bigcup_{i \mid n} \Sigma_{1, \alpha}^{i \mid n}, \quad \text { where } \quad \Sigma_{1, \alpha}^{i \mid n}=\psi_{i \mid n}^{\alpha}\left(\Sigma_{1}^{0}\right)
$$

(see Fig. 2). We proceed in a similar way in order to construct the fiber $\Sigma_{j, \alpha}^{n}, \varepsilon$ neighborhood of $K_{j, \alpha}^{n}(j=2,3)$ and, we define the fiber $\Sigma_{\alpha}^{n}$, $\varepsilon$-neighborhood of $\partial \Omega^{n}$,

$$
\Sigma_{\alpha}^{n}=\bigcup_{j=1}^{3} \Sigma_{j, \alpha}^{n} \quad \text { and } \quad \Omega_{\varepsilon, \alpha}^{n}=\overline{\Omega_{\alpha}^{n}} \bigcup \Sigma_{\alpha}^{n}
$$

From now on, we omit $\alpha$ when it does not give rise to misunderstanding, by writing simply $\Omega$ instead of $\Omega_{\alpha}$ or $\mu$ instead of $\mu_{\alpha}$ and similar expressions. Moreover, we denote by $C$ positive, possibly different constants that do not depend on $n$ and on $\varepsilon$. We note that

$$
\Omega^{n} \subset \Omega^{n+1} \subset \Omega \subset \Omega_{\varepsilon}^{n+1} \subset \Omega_{\varepsilon}^{n} .
$$

We define a weight $w^{n}$ as follows. Let $P$ - for some $i \mid n$ - belong to the boundary $\partial\left(\Sigma_{1}^{i \mid n}\right)$ of $\Sigma_{1, \varepsilon}^{i \mid n}$ and let $P^{\perp}$ be the orthogonal projection of $P$ on $K_{1}^{i \mid n}$. If $x \in \mathbb{R}^{2}$ belongs to the segment with end-points $P$ and $P^{\perp}$, we set, in our current notation,

$$
w_{1}^{n}(x)=\frac{3\left|P-P^{\perp}\right|}{3+b^{2}},
$$

where $\left|P-P^{\perp}\right|$ is the (Euclidean) distance between $P$ and $P^{\perp}$ in $\mathbb{R}^{2}$. We proceed in a similar way in order to construct the weights $w_{j}^{n}$ on $\Sigma_{j}^{n}(j=2,3)$ and we define $w^{n}$ on $\Omega_{\varepsilon}^{n}$

$$
w^{n}(x)=\left\{\begin{array}{lll}
w_{j}^{n}(x) & \text { if } & x \in \Sigma_{j}^{n} \\
1 & \text { if } & x \in \overline{\Omega^{n}}
\end{array}\right.
$$
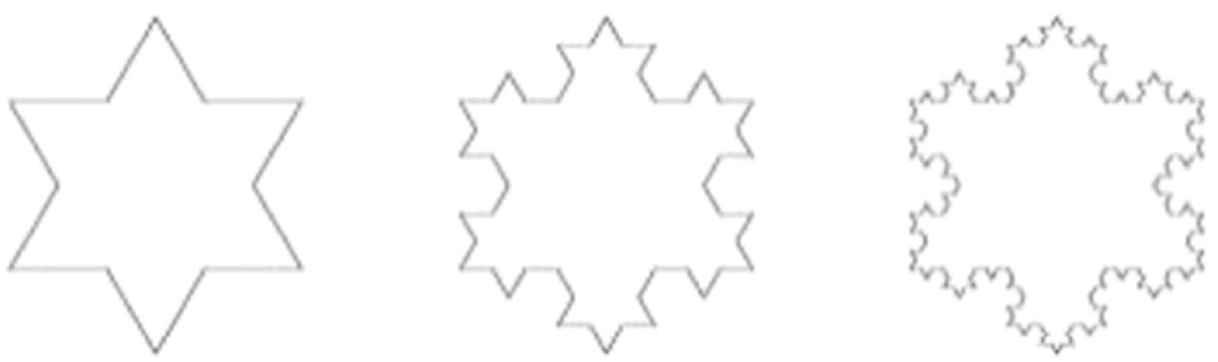

Fig. 1 The pre-fractal domains 

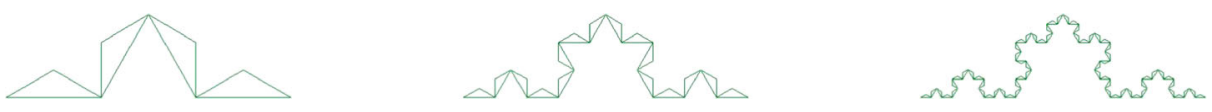

Fig. 2 The fibers

Associated with the weight $w^{n}$, we consider the Sobolev spaces $H^{1}\left(\Omega_{\varepsilon}^{n} ; w^{n}\right)$ and $H_{0}^{1}\left(\Omega_{\varepsilon}^{n} ; w^{n}\right)$, defined as the completion of $C^{\infty}\left(\overline{\Omega_{\varepsilon}^{n}}\right)$ and $C_{0}^{\infty}\left(\Omega_{\varepsilon}^{n}\right)$, respectively, in the norm

$$
\|u\|_{H^{1}\left(\Omega_{\varepsilon}^{n} ; w^{n}\right)}=\left(\int_{\Omega_{\varepsilon}^{n}} u^{2} d x+\int_{\Omega_{\varepsilon}^{n}}|\nabla u|^{2} w^{n} d x\right)^{\frac{1}{2}}
$$

where $d x$ denote the 2-dimensional Lebesgue measure.

We define the coefficients

$$
a_{\varepsilon}^{n}(x)=\left\{\begin{array}{lll}
c_{n} \sigma_{n} w^{n}(x) & \text { if } & x \in \Sigma^{n} \\
1 & \text { if } & x \in \overline{\Omega^{n}}
\end{array}\right.
$$

where

$$
c_{n}>0
$$

and

$$
\sigma_{n}=\frac{\alpha^{n}}{4^{n}}
$$

The following theorem states the existence and the uniqueness of the variational solution of the reinforcement problem. We consider the bilinear form associated with the reinforcement problem

$$
a_{n}(u, v):=\int_{\Omega_{\varepsilon}^{n}} a_{\varepsilon}^{n} \nabla u \nabla v d x+\delta_{n} \int_{\Omega_{\varepsilon}^{n}} u v d x
$$

where $a_{\varepsilon}^{n}$ is defined in Eqs. 2.6, 2.8, 2.7, and $\delta_{n}>0$.

We denote by $\mathfrak{s}$ the arc-length measure on $\partial \Omega^{n}$.

Theorem 1 Let $\sigma_{n}$ be as in Eq. 2.8 and $d_{n} \in \mathbb{R}$. Then, for any $f_{n} \in L^{2}\left(\Omega_{\varepsilon}^{n}\right)$, there exists one and only one solution $u_{n}$ of the following problem

$$
\left\{\begin{array}{l}
\text { find } u_{n} \in H_{0}^{1}\left(\Omega_{\varepsilon}^{n} ; w^{n}\right) \quad \text { such that } \\
a_{n}\left(u_{n}, v\right)=\int_{\Omega_{\varepsilon}^{n}} f_{n} v d x+\sigma_{n} d_{n} \int_{\partial \Omega^{n}} v d \mathfrak{s} \quad \forall v \in H_{0}^{1}\left(\Omega_{\varepsilon}^{n} ; w^{n}\right),
\end{array}\right.
$$

where $a_{n}(\cdot, \cdot)$ is defined in Eq. 2.9. Moreover, $u_{n}$ is the only function that realizes the minimum of the energy functional

$$
\min _{v \in H_{0}^{1}\left(\Omega_{\varepsilon}^{n} ; w^{n}\right)}\left\{a_{n}(v, v)-2 \int_{\Omega_{\varepsilon}^{n}} f_{n} v d x-2 \sigma_{n} d_{n} \int_{\partial \Omega^{n}} v d \mathfrak{s}\right\} .
$$

In the following theorems, we state the existence and uniqueness of the variational solution of the Robin, Neumann, and Dirichlet problems on the domain $\Omega$. We consider the bilinear form associated with the Robin problem

$$
a_{c_{0}}(u, v):=\int_{\Omega} \nabla u \nabla v d x+\delta_{0} \int_{\Omega} u v d x+c_{0} \int_{\partial \Omega} \gamma_{0} u \gamma_{0} v d \mu
$$

where $\mu$ is the measure on $\partial \Omega$ that coincides, on each $K_{j} j=1,2,3$, with the Hausdorff measure (2.2) defined before and $\gamma_{0} u$ denotes the trace of the function $u$ on the boundary of 
$\Omega$, that is for $v$ in $L_{l o c}^{1}(D)$, where $D$ is an arbitrary open set of $\mathbb{R}^{2}$, the trace operator $\gamma_{0}$ is defined as

$$
\gamma_{0} v(P):=\lim _{r \rightarrow 0} \frac{1}{m(B(P, r) \cap D)} \int_{B(P, r) \cap D} v(x) d x
$$

at every point $P \in \bar{D}$ where the limit exists (see, for example, page 15 in [35]). From now on, we suppress $\gamma_{0}$ in the notation, when it does not give rise to misunderstanding, by writing simply $v$ instead of $\gamma_{0} v$ and similar expressions. We assume that

$$
c_{0} \geq 0, \delta_{0} \geq 0, \text { and } \max \left(c_{0}, \delta_{0}\right)>0 .
$$

Theorem 2 Let us assume (2.14) and $d \in \mathbb{R}$. Then, for any $f \in L^{2}(\Omega)$, there exists one and only one solution $u$ of the following problem

$$
\left\{\begin{array}{l}
\text { find } u \in H^{1}(\Omega) \quad \text { such that } \\
a_{c_{0}}(u, v)=\int_{\Omega} f v d x+d \int_{\partial \Omega} v d \mu \quad \forall v \in H^{1}(\Omega)
\end{array}\right.
$$

where $a_{c_{0}}(\cdot, \cdot)$ is defined in Eq. 2.12. Moreover, $u$ is the only function that realizes the minimum of the energy functional

$$
\min _{v \in H^{1}(\Omega)}\left\{a_{c_{0}}(v, v)-2 \int_{\Omega} f v d x-2 d \int_{\partial \Omega} v d \mu\right\} .
$$

In a similar way, we prove the following result. We consider the bilinear form associated with the Dirichlet problem and

$$
a_{\infty}(u, v):=\int_{\Omega} \nabla u \nabla v d x+\delta_{0} \int_{\Omega} u v d x .
$$

We assume that

$$
\delta_{0} \geq 0
$$

Theorem 3 Let us assume (2.18). Then, for any $f \in L^{2}(\Omega)$, there exists one and only one solution $u$ of the following problem

$$
\left\{\begin{array}{l}
\text { find } u \in H_{0}^{1}(\Omega) \quad \text { such that } \\
a_{\infty}(u, v)=\int_{\Omega} f v d x \quad \forall v \in H_{0}^{1}(\Omega)
\end{array}\right.
$$

where $a_{\infty}(\cdot, \cdot)$ is defined in Eq. 2.17. Moreover, $u$ is the only function that realizes the minimum of the energy functional

$$
\min _{v \in H_{0}^{1}(\Omega)}\left\{a_{\infty}(v, v)-2 \int_{\Omega} f v d x\right\} .
$$

We recall the notion of $M$-convergence of functionals, introduced in [41], (see also [42]).

Definition 1 A sequence of functionals $F^{n}: H \rightarrow(-\infty,+\infty]$ is said to $M$-converge to a functional $F: H \rightarrow(-\infty,+\infty]$ in a Hilbert space $H$, if

(a) For every $u \in H$ there exists $u_{n}$ converging strongly to $u$ in $H$ such that

$$
\limsup F^{n}\left[u_{n}\right] \leq F[u], \quad \text { as } n \rightarrow+\infty .
$$

(b) For every $v_{n}$ converging weakly to $u$ in $H$

$$
\liminf F^{n}\left[v_{n}\right] \geq F[u], \quad \text { as } n \rightarrow+\infty .
$$


Let $\Omega^{*}$ be an open regular domain such that $\Omega^{*} \supset \overline{\Omega_{\varepsilon}^{n}}$, for all $n$ : in order to fix notation we choice as $\Omega^{*}$ the ball with the center in the point $P_{0}=\left(\frac{1}{2},-\frac{1}{2}\right)$ and radius 1 . We consider the sequence of weighted energy functionals in $L^{2}\left(\Omega^{*}\right)$

$$
F^{n}[u]= \begin{cases}\int_{\Omega_{\varepsilon}^{n}} a_{\varepsilon}^{n}|\nabla u|^{2} d x+\delta_{n} \int_{\Omega_{\varepsilon}^{n}} u^{2} d x & \text { if }\left.u\right|_{\Omega_{\varepsilon}^{n}} \in H_{0}^{1}\left(\Omega_{\varepsilon}^{n} ; w^{n}\right) \\ +\infty & \text { otherwise in } L^{2}\left(\Omega^{*}\right)\end{cases}
$$

(the coefficients $a_{\varepsilon}^{n}$ are defined in Eqs. 2.6, 2.8, 2.7, $\delta_{n}>0$ ) and

$$
F_{c_{0}}[u]= \begin{cases}\int_{\Omega}|\nabla u|^{2} d x+\delta_{0} \int_{\Omega} u^{2} d x+c_{0} \int_{\partial \Omega} u^{2} d \mu & \text { if }\left.u\right|_{\Omega} \in H^{1}(\Omega) \\ +\infty & \text { otherwise in } L^{2}\left(\Omega^{*}\right) .\end{cases}
$$

Moreover, we consider the case where the layer is weakly insulating (see Eq. 2.31 below) and we introduce the following functional $(2.25)$ in $L^{2}\left(\Omega^{*}\right)$

$$
F_{\infty}[u]= \begin{cases}\int_{\Omega}|\nabla u|^{2} d x+\delta_{0} \int_{\Omega} u^{2} d x & \text { if }\left.u\right|_{\Omega} \in H_{0}^{1}(\Omega) \\ +\infty & \text { otherwise in } L^{2}\left(\Omega^{*}\right) .\end{cases}
$$

In order to study the asymptotic behaviour of the functions $u_{n}$, we fix the further assumptions

$$
\begin{gathered}
f_{n}, f \in L^{2}\left(\Omega^{*}\right), \text { and } f_{n} \rightarrow f \in L^{2}\left(\Omega^{*}\right), \text { as } n \rightarrow+\infty, \\
\delta_{n}>0 \text { and } \delta_{n} \rightarrow \delta_{0} \text { as } n \rightarrow+\infty, \\
c_{n}>0 \text { and } c_{n} \rightarrow c_{0} \text { as } n \rightarrow+\infty, \\
d_{n}, d \in \mathbb{R}, \text { and } d_{n} \rightarrow d \text { as } n \rightarrow+\infty .
\end{gathered}
$$

We also introduce the following results which have been proved in [20] and turn out to be useful further on.

Proposition 1 Let $\sigma_{n}$ be as in Eq. 2.8. Then, for every sequence $g_{n} \in H^{1}(\Omega)$ weakly converging towards $g^{*}$ in $H^{1}(\Omega)$, we have

$$
\sigma_{n} \int_{\partial \Omega^{n}} g_{n} d \mathfrak{s} \rightarrow \int_{\partial \Omega} g^{*} d \mu \text {, as } n \rightarrow+\infty .
$$

Theorem 4 Let us assume (2.28) and (2.27). Then, the sequence of functionals $F^{n}$, defined in Eq. 2.23, $M$-converges in $L^{2}\left(\Omega^{*}\right)$ to the functional $F_{c_{0}}$ defined in Eq. 2.24 as $n \rightarrow+\infty$.

Now we consider the case when the conductivity of the thin fibers vanishes slower than the thickness of the fiber: more precisely, we suppose

$$
c_{n} w^{n} \rightarrow 0, c_{n} \rightarrow+\infty \text {. }
$$

Theorem 5 Let us assume (2.31) and (2.27). Then the sequence of functionals $F^{n}$, defined in Eq. 2.23, $M$-converges in $L^{2}\left(\Omega^{*}\right)$ as $n \rightarrow+\infty$ to the energy functional $F_{\infty}[u]$ defined in Eq. 2.25.

In conclusion, throughout we consider the geometric constant $\sigma_{n}$ as in Proposition 1 and the following condition on the conductivity of the thin fibers $\Sigma^{n}$

$$
c_{n} w^{n} \rightarrow 0 \quad \text { as } n \rightarrow \infty \text {. }
$$




\section{Positive Continuous Additive Functionals and Random Times}

We recall some basic aspects and introduce some notations. Let $E$ be a locally compact separable metric space and $m$ be a positive Radon measure on $E$ such that $\operatorname{supp}[m]=E$. A Dirichlet form $\mathcal{E}$ with domain $D(\mathcal{E})$ is a Markovian closed symmetric form on $L^{2}(E, m)$ (see [30, Chapter 1]). Let $X=\left(\left\{X_{t}\right\}_{t \geq 0} ; \mathfrak{F} ; \mathbb{P}_{x}, x \in E\right)$ be an $m$-symmetric Hunt process whose Dirichlet form $(\mathcal{E}, D(\mathcal{E}))$ on $L^{2}(E, m)$ is regular (see [30, Chapter 5]).

We say that $A_{t}, t \geq 0$ is a positive continuous additive functional (PCAF) and write $A_{t} \in \mathbf{A}_{c}^{+}$denoting by $\mathbf{A}_{c}^{+}$the totality of PCAFs of an $m$-symmetric Hunt process $X$ (see [24, A.3.1] for details). More precisely, we say that $A_{t} \in \mathbf{A}_{c}^{+}$if

A.1) $A_{t}, t \geq 0$ is $\mathcal{F}_{t}$-measurable $\left(\left\{\mathcal{F}_{t}\right\}\right.$ is the minimum completed admissible filtration),

A.2) there exists a set $\Lambda \in \mathcal{F}_{\infty}$ and an exceptional set $N \subset E$ with $\operatorname{Cap}(N)=0$ such that $\mathbb{P}_{x}(\Lambda)=1$ for all $x \in E \backslash N, \theta_{t} \Lambda \subset \Lambda$ for all $t>0$; for every $\omega \in \Lambda, A_{t}(\omega)$ : $t \mapsto A_{t}(\omega)$ is continuous, $A_{0}(\omega)=0$; for all $s, t \geq 0 A_{s+t}(\omega)=A_{t}(\omega)+A_{s}\left(\theta_{t} \omega\right)$ where $\theta_{t}, t \geq 0$ is the (time) translation semigroup,

A.3) for all $\omega \in \bar{\Lambda}, A_{t}(\omega): t \mapsto A_{t}(\omega)$ is non-decreasing.

In this section, we denote by $\mu$ a positive Radon measure on $E$. Hereafter, we write $\langle v, u\rangle_{\mu}=\int_{E} v(x) u(x) \mu(d x)$ and, in some case, we simply write $\langle v, \mu\rangle$ with obvious meaning of the notation. We denote by $C_{0}$ the set of continuous functions with compact support. A positive Radon measure $\mu$ for which ([30, pag. 74])

$$
\int|v(x)| \mu(d x) \leq C \sqrt{\mathcal{E}_{1}(v, v)}, \quad \forall v \in D(\mathcal{E}) \cap C_{0}(E)
$$

where

$$
\mathcal{E}_{\lambda}(u, v)=\mathcal{E}(u, v)+\lambda\langle u, v\rangle_{m}
$$

is said of finite energy integral and formula (3.1) holds if and only if there exists, for each $\lambda>0$, a unique function $U_{\lambda} \mu \in D(\mathcal{E})$ (where $U_{\lambda} \mu$ is a $\lambda$-potential) such that

$$
\mathcal{E}_{\lambda}\left(U_{\lambda} \mu, v\right)=\int v(x) \mu(d x) .
$$

We recall that ([30, pag. 64]), for an open set $B \subset E$ and $\mathcal{L}_{B}=\{v \in D(\mathcal{E}): v \geq$ 1 m-a.e. on $B\}$, the capacity is defined as $\operatorname{Cap}(B)=\inf _{u \in \mathcal{L}_{B}} \mathcal{E}_{1}(u, u)$ if $\mathcal{L}_{B} \neq \emptyset$ and $\operatorname{Cap}(B)=\infty$ if $\mathcal{L}_{B}=\emptyset$. We say that a Borel measure $\mu$ on $E$ is a smooth measure and write $\mu \in S=S(E)$ if ([30, pag. 80])

$\mu .1) \quad \mu$ charges no set of zero capacity;

$\mu .2)$ there exists an increasing sequence $\left\{F_{n}\right\}$ of closed sets such that $\mu\left(F_{n}\right)<\infty$ and $\operatorname{Cap}\left(K \backslash F_{n}\right) \rightarrow 0$ for all compact sets $K$.

The class of smooth measures $S$ is therefore large and it contains all positive Radon measures charging no set of zero capacity. By [30, Lemma 2.2.3], all measures of finite energy are smooth. We use the notation introduced in [30] and denote by $S_{0} \subset S$ the set of positive Radon measure of finite energy integrals, by $S_{00} \subset S_{0}$ the set of finite measures with $\left\|U_{1} \mu\right\|_{\infty}<\infty$.

Let us consider $\mu_{A} \in S$ and $A_{t} \in \mathbf{A}_{c}^{+}$associated with the $m$-symmetric Hunt process $X$ with $\mathbb{P}_{m}(\Lambda)=\int_{E} \mathbb{P}_{x}(\Lambda) m(d x)$ and $\mathbb{P}_{x}(\Lambda)=\mathbb{P}_{x}\left(X_{t} \in \Lambda\right)$ for $\Lambda \in \mathfrak{F}$. Then, the measure 
$\mu_{A}$ and the PCAF $A_{t}$ are in the Revuz correspondence if, for any $f \in \mathcal{B}_{+}(E)$ (the set of non-negative and measurable functions on $E$ ), we have that

$$
\left\langle f, \mu_{A}\right\rangle=\lim _{t \downarrow 0} \frac{1}{t} \mathbb{E}_{m}\left[\int_{0}^{t} f\left(X_{s}\right) d A_{s}\right]=\lim _{\lambda \rightarrow \infty} \lambda \mathbb{E}_{m}\left[\int_{0}^{\infty} e^{-\lambda t} f\left(X_{t}\right) d A_{t}\right] .
$$

We say that $\mu_{A}$ is the Revuz measure of $A \in \mathbf{A}_{c}^{+}$and if $\mu_{A} \in S$, then there exists a unique (up to equivalence) PCAF $\left\{A_{t}\right\}_{t \geq 0}$ with Revuz measure $\mu_{A}$ ([30, Theorem 5.1.4 and Theorem 5.1.3]). Throughout, we write $\mu$ instead of $\mu_{A}$ if no confusion arises. Moreover, we introduce

$$
R_{\lambda} f(x)=\mathbb{E}_{x}\left[\int_{0}^{\infty} e^{-\lambda t} f\left(X_{t}\right) d t\right] \quad \text { and } \quad U_{A}^{\lambda} f(x)=\mathbb{E}_{x}\left[\int_{0}^{\infty} e^{-\lambda t} f\left(X_{t}\right) d A_{t}\right]
$$

(see [47] for a complete discussion). Throughout, we consider the local time process $L_{t}^{\partial E}=$ $L_{t}^{\partial E}(X)=\int_{0}^{t} \mathbf{1}_{\partial E}\left(X_{s}\right) d s$ which is the PCAF increasing when $X$ hits the boundary $\partial E$.

We introduce some further notation and basic aspects. In the following sections we consider the killed process

$$
X_{t}= \begin{cases}\widetilde{X}_{t}, & t<\tau \\ \partial, & t \geq \tau\end{cases}
$$

( $\widetilde{X}_{t}$ admits no killing inside $E$ and $\partial$ is the "coffin state" not in $E$ ) where $\tau$ will be a suitable random time and $\mathbf{P}_{t} f(x)=\mathbb{E}_{x}\left[f\left(X_{t}\right)\right]=\mathbb{E}_{x}\left[f\left(\widetilde{X_{t}}\right) ; t<\tau\right], x \in E$ is the associated semigroup. In particular, we consider the following cases: i) $\tau=\zeta^{E}$ is a random time such that $\left(\zeta^{E}<t\right) \equiv\left(L_{t}^{\partial E}>\zeta\right)$ and $\zeta$ is an exponential random variable, with parameter $c_{0} \in(0, \infty)$, independent from $X$; ii) $\tau=\infty$ under suitable conditions; iii) $\tau=\tau_{E}$ is the exit time of $X$ from $E$.

Thus $X_{t}, t \in[0, \infty]$, is a Markov process with state space $E_{\partial}:=E \cup\{\partial\}$. The transition function is not conservative according with the cemetery point $\{\partial\}$, that is $\mathbb{P}_{x}\left(X_{t}=\partial\right) \geq 0$, $\forall x \in E_{\partial}, t \geq 0$. In particular, $X$ is conservative if $\mathbb{P}_{x}\left(\zeta^{E}<\infty\right)=0$ for every $x \in E$ where we denote by $\zeta^{E}$ also the lifetime of the process on $E$. Since $X_{t}$ is a Markov process, $\mathbb{P}_{x}\left(X_{0}=x\right)=1$ for all $x \in E_{\partial}$ and $\mathbb{P}_{\partial}\left(X_{t}=\partial\right)=1$ for all $t$. Our discussion is mainly concerned with trap domains. A point $x \in E_{\partial}$ is called a trap of $X$ if $\mathbb{P}_{x}\left(X_{t}=x\right)=1$ for every $t \geq 0$. We give the definition of trap domain further on in the text. In i) it is well known that, the lifetime of the process follows the law $\mathbb{P}_{x}\left(\zeta^{E}>t \mid X_{t}\right)=e^{-c_{0} L_{t}^{\partial E}}$ for every $x \in E$ and $t>0$. Thus, $L_{0}^{\partial E}=0$ and $\mathbb{P}_{x}\left(\zeta^{E}>0\right)=1$. $L^{\partial E}$ is the occupation time of $X$ on $\partial E$. For $\Lambda \subseteq E$, we denote by $\Gamma_{t}^{\Lambda}(X)=\operatorname{meas}\left\{s \in[0, t]: X_{s} \in \Lambda\right\}$ the occupation time process of $X$ on $\Lambda$. The semigroup $\mathbf{P}_{t}$ is strongly continuous and we use the fact that $\lambda R_{\lambda} f \rightarrow f$ and $\lambda\left\langle U_{A}^{\lambda} f, m\right\rangle \rightarrow\langle f, \mu\rangle$ as $\lambda \rightarrow \infty$ where $\mu$ is the Revuz measure associated with the additive functional $A$ and therefore, to the random time $\tau \in\left[\tau_{E}, \infty\right]$.

Let us consider the perturbed Dirichlet form on $L^{2}(E, m)$ written as

$$
\mathcal{E}_{\lambda}^{\mu}(u, v)=\mathcal{E}_{\lambda}(u, v)+\langle u, v\rangle_{\mu}, \quad u, v \in D(\mathcal{E}) \cap L^{2}(E, \mu)
$$

where $\mathcal{E}_{\lambda}$ has been introduced in Eq. 3.2, $\mu \in S$. Let $A_{t} \in \mathbf{A}_{c}^{+}$and $\widetilde{X_{t}}$ as in Eq. 3.6. The transition function

$$
\mathbf{P}_{t}^{\mu} f(x)=\mathbb{E}_{x}\left[e^{-A_{t}} f\left(\widetilde{X_{t}}\right)\right]
$$

is associated with the regular form $\left(\mathcal{E}_{0}^{\mu}, D\left(\mathcal{E}_{0}^{\mu}\right)\right.$ ) where $\mu$ is the Revuz measure of $A_{t}$ (see [30, Theorem 6.1.1 and Theorem 6.1.2]). We simply write $\mathbf{P}_{t}$ instead of $\mathbf{P}_{t}^{\mu}$. In the following 
sections we consider $m$-version of $\tilde{X}$ associated with our problems on fractal domains (and pre-fractal if clearly specified).

We say that $X^{n}$ converges in law to $X$ and write $X^{n} \stackrel{\text { law }}{\rightarrow} X$ if $\mathbb{E} f\left(X^{n}\right) \rightarrow \mathbb{E} f(X)$ as $n \rightarrow \infty$ for every continuous and bounded function $f$.

Throughout, we consider the PCAF (in the strict sense, that is, in A.2) $\Lambda$ is the defining set and $N$ is an empty set) $A_{t}^{n}=\int_{0}^{t} f\left(X_{s}^{n}\right) d s$, the multiplicative functional $M_{t}^{n}=e^{-A_{t}^{n}}$ and a stopping time $T_{n}$. We have that (see [23, Lemma 2.1])

$$
\lim _{t \downarrow 0} \mathbb{E}_{x}^{n} A_{t}^{n}=\lim _{t \downarrow 0}\left[\mathbb{E}_{x}^{n}\left[A_{t}^{n} ; t<T_{n}\right]+\mathbb{E}_{x}^{n}\left[A_{t}^{n} ; t \geq T_{n}\right]\right]=\lim _{t \downarrow 0} \mathbb{E}_{x}^{n}\left[A_{t}^{n} ; t<T_{n}\right] .
$$

\section{Transmission Condition on Regular Interfaces}

In this section we consider the probabilistic approach of thin layer when $\Omega$ is a disc. Actually, we provide a sketch of proof for the problem with collapsing annulus by following two approaches. Here, the purpose is to underline the main differences with the fractal case investigated in the next sections. Notice also that speed measure and scale function characterize uniquely one-dimensional diffusions.

First Approach Let us consider a BM $X$ on $\mathbb{R}^{2}$ started (at $x \in \mathbb{R}^{2}$ ) away from zero. For $\theta^{\prime} \in[0,2 \pi), r^{\prime}>0$ we can write, $\mathbb{P}_{x}\left(X_{t} \in d y\right)=\mathbb{P}_{\left(\theta^{\prime}, r^{\prime}\right)}\left(\Theta_{t} \in d \theta, R_{t} \in d r\right)$ where $R=|X|$ is a Bessel process. In particular, $R$ and $\Theta$ are the radial and the angular part of $X$. It is also well-known that a skew-product representation is given in term of $(R, \Theta)$ where $R=|X|$ is a Bessel process and $\Theta=X /|X|=B\left(\int R_{z}^{-2} d z\right)$ with $B$ an independent BM on the sphere $\mathbb{S}^{1}$ ([34, pag. 269]). Here $\Theta$ is a time-changed BM on $\mathbb{S}^{1}$ (the winding number of $\left.X_{t}\right)$.

Let $v \in(0,1)$ and $B^{v}$ be a planar BM on the disc $C_{2}$ with a disc $C_{1} \subset C_{2}$ (centred at the same point $(0,0)$, with radius $\left.r_{1}<r_{2}=r_{1}+\varepsilon, \varepsilon>0\right)$, Dirichlet condition on $\partial C_{2}$ and transmission condition on $\partial C_{1}$ (the skew condition, that is $\mathbb{P}_{x}\left(B_{t}^{v} \in C_{2} \backslash \overline{C_{1}}\right)=v$, $\mathbb{P}_{x}\left(B_{t}^{v} \in C_{1}\right)=1-v$ for $\left.x \in \partial C_{1}\right)$. Due to the non-symmetry $(1-v, v)$ we say that $B^{v}$ is a skew planar BM (that is a 2-dimensional extension of the skew BM, see for example Section 11.10 of [39] or [52]). Let $\mathcal{L}_{n}$ be the governing operator of $B^{\nu}$. We examine in this section the classical case corresponding to the (formal) problem

$$
\begin{aligned}
\mathcal{L}_{n} u_{n} & =-f_{n} \quad \text { on } C_{2} \\
\left.(1-v) \partial_{\mathbf{n}} u_{n}\right|_{\partial C_{1}-} & =\left.v \partial_{\mathbf{n}} u_{n}\right|_{\partial C_{1}+} \\
\left.u_{n}\right|_{\partial C_{1}-} & =\left.u_{n}\right|_{\partial C_{1}+} \\
\left.u_{n}\right|_{\partial C_{2}} & =0
\end{aligned}
$$

where $\partial_{\mathbf{n}}$ is the normal derivative and we denote by $\partial C_{1}-$ and $\partial C_{1}+$ the boundary from the interior and from the exterior of $C_{1}$. Let us consider the sequences $v=v(n), \varepsilon=\varepsilon(n)$, $n \in \mathbb{N}$. Our aim is to study the asymptotic behaviour of the solution as $n \rightarrow \infty$ and $\nu, \varepsilon \rightarrow 0, v / \varepsilon \rightarrow c$ with different rate given by the elastic coefficient $c \geq 0$. Then, the problem above can be associated with $B^{v}$ started away from the origin, that is the process is partially (normally) reflected on $\partial C_{1}$ and totally absorbed in $\partial C_{2}$.

A reflecting $\mathrm{BM}$ on a disc can be constructed (in law) by considering suitable time change and rotation ([34, pag. 272]). The time change in this case is a stochastic clock given by an additive functional of the radial motion as indicated before. Denote by $C_{1,2}$ the annu- 
lus $C_{2} \backslash \overline{C_{1}}$. Thus, for $0<r^{\prime}<r_{2}$ and $0<\theta^{\prime} \leq 2 \pi, \mathbb{P}_{x}\left(B_{t}^{v} \in d y\right)=\mathbb{P}_{\left(\theta^{\prime}, r^{\prime}\right)}\left(\Theta_{t} \in d \theta, R_{t}^{v} \in\right.$ $d r)$ where $R^{v}=\left|B^{v}\right|$ is a skew Bessel process on $\left(0, r_{2}\right)$ such that, $\mathbb{P}_{r_{1}}\left(R_{t}^{v} \in\left(r_{1}, r_{2}\right)\right)=v$. We have normal reflection on $\partial C_{1}$ and

$$
\forall x \in \partial C_{1}, \quad \mathbb{P}_{x}\left(B_{t}^{v} \in d y\right)=\frac{d \theta}{2 \pi} \mathbb{P}_{r_{1}}\left(R_{t}^{v} \in d r\right) .
$$

The BM can move from $\partial C_{1}$ according with an uniformly distributed angle $\Theta$ for the choice of the starting point, that is

$$
\int_{0}^{2 \pi} \mathbb{P}_{\left(\theta, r_{1}\right)}\left(B_{t}^{\nu} \in C_{1,2}\right) \frac{d \theta}{2 \pi}=v \quad \text { and } \quad \int_{0}^{2 \pi} \mathbb{P}_{\left(\theta, r_{1}\right)}\left(B_{t}^{\nu} \in C_{1}\right) \frac{d \theta}{2 \pi}=1-v .
$$

Let $R$ be the part of the Bessel process $\widetilde{R}$ on $\left(0, r_{2}\right)$ with $\widetilde{R} \in(0, \infty)$. We cut the excursions of $\widetilde{R}$ by considering a time change given by the inverse of $\Gamma_{t}^{\left(r_{1}, r_{2}\right)}(\widetilde{R})$. We do the same with $\Gamma_{t}^{\left(0, r_{1}\right)}(\widetilde{R})$. As in [34, pag. 115] we can obtain a skew motion by considering the $v$ portion of $\Gamma_{t}^{\left(r_{1}, r_{2}\right)}(\widetilde{R})$ and the $1-v$ portion of $\Gamma_{t}^{\left(0, r_{1}\right)}(\widetilde{R})$, that is a new occupation time, say $\mathfrak{f}$. Thus, it is possible to consider a suitable time change $\mathfrak{f}^{-1}$, in order to obtain partial (normal) reflection on $r_{1}$ and, $R^{v}=R_{\mathfrak{f}^{-1}}$ is a Bessel process on $\left(0, r_{2}\right)$ with transmission condition on $r_{1}$. The skew BM constructed in this way has the skew-product representation involving the time-changed Bessel process $R_{\mathfrak{f}^{-1}}$ where the $\mathrm{BM}$ on the circle is identical in law to the original process (that is, $\Theta \stackrel{\text { law }}{=} \Theta_{\mathfrak{f}^{-1}}$ ). More precisely, let us consider $T_{t}^{\nu}=\int_{0}^{\mathfrak{f}^{-1}(t)}\left(R_{z}^{\nu}\right)^{-2} d z$ where $R_{t}^{v}=R_{\mathfrak{f}^{-1}(t)}$ and $T_{t}=\int_{0}^{t} R_{z}^{-2} d z$. Then $\Theta_{t}^{v}=B\left(T_{t}^{\nu}\right)$ where $B$ is independent from $T_{t}^{v}$ and $\Theta_{t}=B\left(T_{t}\right)$ where $B$ is independent from $T_{t}$. Since $T_{t}^{\nu} \stackrel{\text { law }}{=} T_{t}$ we get that $\Theta_{t}^{v} \stackrel{\text { law }}{=} \Theta_{t}$.

Thus, the only process we consider is the radial part $R_{t}$ time-changed by $\mathfrak{f}^{-1}$, that is $R^{v}$. The Bessel process can start from zero and then it is instantaneously reflected. It never hits the origin at some $t>0$. The mean exit time

$$
v_{\varepsilon}(r)=\mathbb{E}\left[\tau_{\left(0, r_{2}\right)}\left(R^{v}\right) \mid R_{0}^{v}=r \in\left(0, r_{2}\right)\right]=\mathbb{E}_{r} \tau_{C_{2}}
$$

can be explicitly written by following standard techniques for one-dimensional diffusions (see for example [37]) and, as $\varepsilon \rightarrow 0, v \rightarrow 0$ according with $v / \epsilon \rightarrow c$, we find that it solves

$$
\begin{array}{ll}
v_{0}^{\prime \prime}=-1 & \\
v_{0}(0)=0 & \text { if } c=\infty \\
v_{0}\left(r_{1}\right)=0 & \\
v_{0}^{\prime}\left(r_{1}\right)=-c v_{0}\left(r_{1}\right) & \text { if } c \in[0, \infty) .
\end{array}
$$

This corresponds to the study of $u_{n}$ with $f_{n}=\mathbf{1}$. Due to isotropy and the discussion about the angular part of the planar BM, we arrive at the solution $u_{\infty}$ of the problem above. Therefore, the boundary conditions on $r_{1}$ depend on the limit of the ratio between the skewness coefficient $v$ and the thickness coefficient $\varepsilon$. According with Section 2, we note that $\sigma_{n}=1 / 2 \pi, w^{n}=\varepsilon(n)$ and $c_{n} w^{n}=v(n), \Sigma^{n}=C_{1,2}$ is the thin layer.

Second Approach Alternatively, we can approach the problem as follows. Let $T_{c_{n}}$ be the stopping time for the skew BM on $C_{2}$ with $r_{2}=r_{1}+\varepsilon(n)$ and $v=v(n)$ under the assumption that $\lim _{n \rightarrow \infty} c_{n}=\lim _{n \rightarrow \infty} v(n) / \varepsilon(n)$. The lifetime depends on the asymptotic behaviour of the process on the collapsing annulus $C_{1,2}$. Our result in fractal domains can be reformulated here (in regular domains) by considering the stopping time $T_{C_{n}}$ and the 
fact that $\left(T_{c_{n}}>t \mid B^{v}\right) \equiv\left(T_{c_{n}}>t \mid R^{v}\right)$ in view of the previous discussion about $\Theta \stackrel{\text { law }}{=}$ $\Theta_{\mathfrak{f}^{-1}}$. In particular we consider the lifetime $\zeta^{C_{2}}=T_{c_{n}}$ of $R^{v}$ and $\widehat{\zeta^{C_{2}}}=\tau_{C_{2}} \wedge \widehat{T_{c_{n}}}$ where $\widehat{T_{c_{n}}}=\inf \left\{s>0: L_{s}^{r_{1}}>\zeta^{n}\right\}$ with conditional law $\mathbb{P}_{x}\left(\widehat{T_{c_{n}}}>t \mid R^{v}\right)=\exp -c_{n} L_{t}^{r_{1}}$ where $L_{t}^{r_{1}}$ is the symmetric local time of $R^{v}$ at $r_{1}$. That is, we consider $\zeta^{n}$ as exponential random variable with parameter $c_{n}$ and independent from $R^{v}$. Thus, under the assumption that $\lim _{n \rightarrow \infty} c_{n}=\lim _{n \rightarrow \infty} v(n) / \varepsilon(n)$, we study the asymptotic behaviour of

$$
u_{n}(r)=\mathbb{E}_{r}\left[\int_{0}^{T_{c_{n}}} f_{n}\left(\widetilde{R_{t}^{v}}\right) d t\right]=\mathbb{E}_{r}\left[\int_{0}^{\infty} f_{n}\left(R_{t}^{\nu}\right) M_{t}^{n} d t\right]
$$

where $M_{t}^{n}=\mathbf{1}_{\left(t<T_{c_{n}}\right)}$ by means of the asymptotic behaviour of

$$
\widehat{u_{n}}(r)=\mathbb{E}_{r}\left[\int_{0}^{\infty} f_{n}\left(R_{t}^{v}\right) \widehat{M_{t}^{n}} d t\right]
$$

where $\widehat{M_{t}^{n}}=\mathbf{1}_{\left(t<\widehat{T_{c_{n}}}\right)}$ and (assume here $x \in C_{1}$ for the reader's convenience)

$$
\mathbb{P}_{x}\left(\widehat{T_{c_{n}}}>t \mid R^{v}\right) \stackrel{\text { law }}{\rightarrow}\left\{\begin{array}{ll}
\mathbf{1}, & c_{n} \rightarrow 0, \\
\exp -c_{0} L_{t}^{r_{1}}, & c_{n} \rightarrow c_{0} \\
\mathbf{1}_{\left(t<\tau_{C_{1}}\right)}, & c_{n} \rightarrow \infty,
\end{array} \quad \text { as } n \rightarrow \infty .\right.
$$

For the local times we have that $L_{t}^{r_{1}}\left(R^{v(n)}\right) \rightarrow L_{t}^{r_{1}}\left(R^{+}\right)$in law where $R^{+}$is a reflecting Bessel process on $\left(0, r_{1}\right)$. Thus, we estimate the stopping time $T$ by $\widehat{T}$ and exploit the fact that $\widehat{\zeta^{C_{2}}} \leq \zeta^{C_{2}}$ with probability one. This immediately follows by considering the definition of $\widehat{\zeta^{C_{2}}}$ which can be also written as $\widehat{\zeta^{C_{2}}}=\inf \left\{s \in\left(0, \zeta^{C_{2}}\right]: L_{s}^{r_{1}}>\zeta^{n}\right\}$. The convergence of $R^{v(n)}$ can be obtained by considering that $\mathbb{P}_{r}\left(R_{t}^{\nu(n)}>M\right) \leq M^{-1} \mathbb{E} R_{t}^{\nu(n)}$ and that the moment is bounded.

Remark 1 For a compact subset $K \subset \mathbb{R}^{d}([48$, Theorem 22.7])

$$
\mathbb{P}_{x}\left(B_{t} \in K \text { for some } t>0\right)=\int G(x, y) \mu_{K}(d y)=G \mu_{K}(x)
$$

is a potential of a unique measure $\mu_{K}$ concentrated on $\partial K$. The capacity $\operatorname{Cap}(K)=$ $\inf \{\mathcal{E}(\mu): G \mu \geq 1$ on $K\}$ where $\mathcal{E}(\mu)=\int G(x, y) \mu(d x) \mu(d y)$ can be defined from $\mu_{K}(K)$.

Define $\sigma_{K}=\sup \left\{s>0 ; B_{s} \in K\right\}$ with $\sup \emptyset=0$, then for $x \in \mathbb{R}^{d}, y \in K, t>0$, we have that $([25,31])$

$$
\mathbb{P}_{x}\left(B_{\sigma_{K}} \in d y, \sigma_{K} \in d t\right)=p(t, x, y) \mu_{K}(d y) d t
$$

and we recover an interesting connection between elastic coefficient and capacity. Consider $K=\overline{C_{1}}$ : the last exit time can be therefore rewritten as $\sigma_{K}=\inf \left\{s>0: L_{s}^{r_{1}}>\zeta^{n}\right\}$ where now $\zeta^{n}$ is the time the process spends on (or cross) $r_{1}$ before absorption in $r_{2}$.

Remark 2 Notice that we used isotropy and skew product representation which are not suitable tools for approaching our fractal problem. In particular, if we consider the Koch domain $\Omega$, the normal vector does not exist at almost all boundary points. However it is possible to define the Robin boundary condition in the sense of the dual of certain Besov spaces (see [17, Theorem 4.2 ]). 


\section{Transmission Conditions on Irregular Interfaces}

In this section we introduce the modified skew BM $B_{t}^{v, *}, t \geq 0$ on $\Omega_{\varepsilon}^{n}$. The parameter $v \in[0,1]$ is the so called skewness parameter. Skew BM is a process with associated Dirichlet form in $L^{2}\left(\Omega_{\varepsilon}^{n}, \mathfrak{m}_{v}\right)$ given by

$$
\mathcal{E}(u, v)=\frac{1}{2} \int_{\Omega_{\varepsilon}^{n}} \nabla u \nabla v d \mathfrak{m}_{v}, \quad D(\mathcal{E})=H^{1}\left(\Omega_{\varepsilon}^{n}, \mathfrak{m}_{v}\right)
$$

where $\mathfrak{m}_{v}(x)=2(1-v) \mathbf{1}_{\Omega^{n}}(x)+2 v \mathbf{1}_{\Sigma^{n}}(x)$ and it can be associated with discontinuous diffusion coefficients. We focus on the sequence of elliptic operators

$$
L_{n} u=-\operatorname{div}\left(a_{\varepsilon}^{n} \nabla u\right)
$$

in divergence form with coefficients given in Eq. 2.6 and

$$
D\left(L_{n}\right)=\left\{u \in L^{2}\left(\Omega_{\varepsilon}^{n}, d x\right),:\left.u\right|_{\Omega^{n}} \in H^{2}\left(\Omega^{n}\right),\left.\quad u\right|_{\Sigma^{n}} \in H^{2}\left(\Sigma^{n}\right)\right\} .
$$

The discontinuous coefficients $a_{\varepsilon}^{n}$ in Eq. 5.2 introduce the transmission condition in the $L^{2}\left(\partial \Omega^{n}\right)$

$$
\left.\nabla u \cdot \mathbf{n}\right|_{y-}=\left.c_{n} \sigma_{n} \nabla u \cdot \mathbf{n}\right|_{y+} \quad \forall y \in \partial \Omega^{n}
$$

(where $\mathbf{n}$ is the outer normal to $\Omega^{n}, y^{-}=y \in \overline{\Omega^{n}} \cap \partial \Omega^{n}$ and $y^{+}=y \in \overline{\Sigma^{n}} \cap \partial \Omega^{n}$, we recall that $\left.\left.w^{n}\right|_{\partial \Omega^{n}}=1\right)$ and therefore, the corresponding diffusion behaves like a skew BM. For a given $n$, the operator (5.2) can be regarded as the governing operator of the planar skew BM $\widetilde{B^{v}}=\left(\left\{\widetilde{B_{t}^{v}}\right\}_{t \geq 0} ; \mathfrak{F}^{v} ; \mathbb{P}_{x}^{n}, x \in \Omega_{\varepsilon}^{n}\right)$ on $\mathbb{R}^{2}$ from which we define the killed process $B^{\nu}$. Let $\mathcal{L}_{n}$ be the governing operator of $B^{\nu}$ on $L^{2}\left(\Omega_{\varepsilon}^{n}, d x\right)$ with

$$
\begin{array}{r}
\mathcal{D}\left(\mathcal{L}_{n}\right)=\left\{u \in L^{2}\left(\Omega_{\varepsilon}^{n}, d x\right),\left.\quad u\right|_{\Omega^{n}} \in H^{2}\left(\Omega^{n}\right),\left.\quad u\right|_{\Sigma^{n}} \in H^{2}\left(\Sigma^{n}\right),\right. \\
\left.\left.u\right|_{\partial \Omega_{\varepsilon}^{n}}=0, \quad u \text { is continuous on } \partial \Omega^{n} \text { and satisfies (5.3) }\right\} .
\end{array}
$$

Then, the transition function $\mathbf{P}_{t}^{n} f(x)=\mathbb{E}_{x}^{n}\left[f\left(B_{t}^{\nu}\right)\right]=\mathbb{E}_{x}^{n}\left[f\left(\widetilde{B_{t}^{\nu}}\right) ; t<\tau_{\Omega_{\varepsilon}^{n}}\right]$ with transition kernel $p^{v}$ where $v$ depends on the coefficients $a_{\varepsilon}^{n}$ and therefore, on Eq. 5.3, is governed by

$$
\frac{\partial u}{\partial t}=\mathcal{L}_{n} u \quad \text { on } \quad \Omega_{\varepsilon}^{n}
$$

and $\mathcal{L}_{n} f:=\frac{1}{2} L_{n} f, f \in \mathcal{D}\left(\mathcal{L}_{n}\right)$. The parabolic equation (5.4) can be rewritten by considering the infinitesimal generator $\widetilde{\mathcal{L}}_{n}:=\frac{1}{2} \Delta$ on $L^{2}\left(\widetilde{\mathfrak{m}}_{\mathrm{v}}\right)$ with $\mathcal{D}\left(\widetilde{\mathcal{L}}_{n}\right)=\mathcal{D}\left(\mathcal{L}_{n}\right)$ (see [24, pag 356] for details) where

$$
\widetilde{\mathfrak{m}}_{v}(x)=\mathbf{1}_{\Omega^{n}}(x)+c_{n} \sigma_{n} w^{n} \mathbf{1}_{\Sigma^{n}}(x) .
$$

From the transition kernel $p^{v}$ we can write

$$
\mathbb{P}_{x}^{n}\left(\widetilde{B_{t}^{v}} \in \Lambda, t<\tau_{\Omega_{\varepsilon}^{n}}\right)=\int_{\Lambda} p^{v}(t, x, y) d y \quad x \in \Omega_{\varepsilon}^{n}
$$

for some Borel set $\Lambda \in \mathfrak{F}^{v}$ with the (first) exit time

$$
\tau_{\Omega_{\varepsilon}^{n}}=\inf \left\{s>0: \widetilde{B_{s}^{v}} \notin \Omega_{\varepsilon}^{n}\right\} .
$$

We refer to $B^{v}$ as a modified skew BM in the sense that it depends on both the skewness coefficient $v$ (that is the BM is skew) and the weight $w^{n}$ given in Eq. 2.4 (that is, the skew BM is modified). The process $B^{v}$ represents a Brownian diffusion of a particle with transmission condition (5.3) on the pre-fractal $\partial \Omega^{n}$. The BM is partially reflected when it 
hits $\partial \Omega^{n}$ : that is, $\forall x \in \partial \Omega^{n}$ the process starting from $x$ moves toward $\Omega^{n}$ or $\Sigma^{n}$ with probability $1-v$ or $v$ respectively by taking into account the structural constant $\sigma_{n}$. In particular, according with Eq. 4.2,

$$
\sigma_{n} \int_{\partial \Omega^{n}} \mathbb{P}_{x}^{n}\left(B_{t}^{v} \in \Sigma^{n}\right) d \mathfrak{s}=v \quad \text { and } \quad \sigma_{n} \int_{\partial \Omega^{n}} \mathbb{P}_{x}^{n}\left(B_{t}^{v} \in \Omega^{n}\right) d \mathfrak{s}=1-v .
$$

Let $v=v(n)$ be a sequence such that $v(n) \rightarrow 0$ as $n \rightarrow \infty$. Heuristically, Eqs. 5.8 and 5.3 say that

$$
\frac{a_{\varepsilon}^{n+}}{a_{\varepsilon}^{n-}+a_{\varepsilon}^{n+}} \frac{1}{v(n) \sigma_{n}} \rightarrow 1 \quad \text { uniformly on } \partial \Omega^{n} \text { as } n \rightarrow \infty
$$

Since condition (2.32) holds true, from the construction we present here, it must be that $v(n) / c_{n} w^{n} \rightarrow 1$ on $\Sigma^{n}$ as $n \rightarrow \infty$. Equivalently,

$$
\frac{v(n)}{1-v(n)} \frac{1}{c_{n} w^{n}} \rightarrow 1 \quad \text { uniformly on } \Sigma^{n} \text { as } n \rightarrow \infty .
$$

In view of Eq. 5.9, we also refer to $v$ as transmission parameter. However, due to the fact that $\left.w^{n}\right|_{\partial \Omega^{n}}=1$, we must pay particular attention on the pre-fractal boundary.

We follow the characterization of trap domain given in $[8,15]$. Consider an open connected set $D \subset \mathbb{R}^{d}, d \geq 2$ with finite volume and the reflected BM $B^{+}$on $\bar{D}$. Let $\mathcal{B} \subset D$ be an open ball with non-zero radius and denote by $\tau_{\partial \mathcal{B}}=\inf \left\{s \geq 0: B_{s}^{+} \in \partial \mathcal{B}\right\}$ the hitting time of the reflecting $\mathrm{BM} B^{+} \in D \backslash \mathcal{B}$.

Definition 2 The set $D$ is a trap domain if

$$
\sup _{x \in D \backslash \mathcal{B}} \mathbb{E}_{x} \tau_{\partial \mathcal{B}}=\infty
$$

Otherwise, $D$ is a non-trap domain.

Notice that the definition above does not depend on the choice of $\mathcal{B}$ ([15, Lemma 3.3]). In both Lipschitz domains $\Omega^{n}$ and $\Sigma^{n}$ the process $B^{v}$ behaves like a BM $B^{+}$reflecting on $\partial \Omega^{n}$. As shown in $[8,15]$, the pre-fractal and fractal Koch domains are non-trap. Then, $\forall n$, $\Omega^{n}$ and $\Sigma^{n}$ are non trap for $B^{\nu}$. Condition (5.10) can be rewritten in analytic way as follows

$$
\sup _{x \in D \backslash \mathcal{B}} \int_{D \backslash \mathcal{B}} G^{+}(x, y) d y=\infty
$$

where $G^{+}$is the Green function of $B^{+}$on $D$ and $D=\Omega^{n}$ or $D=\Sigma^{n}$.

The process $B^{v}$ on $\Omega_{\varepsilon}^{n}$ is a transient BM for which $\mathbf{P}_{t}^{n} \mathbf{1}_{\Omega_{\varepsilon}^{n}}(x)=\mathbb{P}_{x}\left(\tau_{\Omega_{\varepsilon}^{n}}>t\right)$ and $\mathbb{E}_{x} \tau_{\Omega_{\varepsilon}^{n}}=\int \mathbf{P}_{t}^{n} \mathbf{1}_{\Omega_{\varepsilon}^{n}}(x) d t<\infty$. Nevertheless, we are looking for asymptotic results concerning also a non transient limit process. Thus, for the skew BM $B_{t}^{v}, t<\tau_{\Omega_{\varepsilon}^{n}}$, we introduce the Green function $G_{n}^{v}(x, y)=\int_{0}^{\infty} e^{-\delta_{n} t} p^{v}(t, x, y) d t$ for which we write

$$
G_{n}^{v} f(x)=\int G_{n}^{\nu}(x, y) f(y) d y=\mathbb{E}_{x}^{n}\left[\int_{0}^{\tau_{\Omega_{\varepsilon}^{n}}} e^{-\delta_{n} t} f\left(\widetilde{B_{t}^{\nu}}\right) d t\right]
$$

where $\mathbb{E}_{x}^{n}$ is the expectation under (5.6). Furthermore, we write

$$
G_{n}^{v} f(x)=\int G_{n}(x, y) f(y) \mathfrak{m}_{v}(y) d y
$$

where $G_{n}(\cdot, \cdot)=\int_{0}^{\infty} p(t, \cdot, \cdot) d t$ is the Green function of a BM $B$ on $\Omega_{\varepsilon}^{n}$. 
Let us consider the reflected Brownian motions $B$ on $\Omega^{n}$ and $B^{w}$ on $\mathbb{R}^{2} \backslash \overline{\Omega^{n}}$ where $B^{w}$ and $B$ are independent Brownian motions with $\mathbb{P}_{x}\left(B_{t}^{w} \in \mathbb{R}^{2} \backslash \overline{\Omega^{n}}\right)=1$ and $\mathbb{P}_{x}\left(B_{t} \in\right.$ $\left.\Omega^{n}\right)=1$ and depending on $w^{n}$ with $w^{n} \neq 1$ only outside $\overline{\Omega^{n}}$. We introduce the following representation

$$
\widetilde{B_{t}^{v}}:=\left\{\begin{array}{lll}
B_{t}^{w} & \text { on } \mathbb{R}^{2} \backslash \overline{\Omega^{n}} & \text { with probability } v \\
B_{t} & \text { on } \Omega^{n} & \text { with probability } 1-v
\end{array} .\right.
$$

Thus, $\widetilde{B_{t}^{v}} \stackrel{\text { law }}{=} \widetilde{B_{t}^{v}}$, that is $\widetilde{B_{t}^{v}}$ equals $B_{t}^{w}$ with probability $v$ and $B_{t}$ with probability $1-v$.

We now introduce the process $\widetilde{B_{t}^{v}, *}$ which is the $m$-symmetric extension of $\widetilde{B_{t}^{v}}$ to $\left(\mathbb{R}^{2} \backslash\right.$ $\left.\Omega^{n}\right) \cup \overline{\Omega^{n}}$ (see [53, Remark 1.1], [24, Definition 7.5.8 and Definition 7.7.1]). To be precise, we say that $\widetilde{B_{t}^{v, *}}$ is an $m$-symmetric extension meaning that

$$
m\left(\partial \Omega^{n}\right)=0
$$

where $m$ is the 2-dimensional Lebesgue measure. The process $B^{v, *}$ is the part process of $\widetilde{B_{t}^{v, *}}$ on $\Omega_{\varepsilon}^{n}$ where $\widetilde{B_{t}^{v, *}}$ equals $B^{*}$ on $\overline{\Omega^{n}}$ (the $m$-symmetric extension of $B$ on $\overline{\Omega^{n}}$ ) and $\widetilde{B_{t}^{v, *}}$ equals $B^{w, *}$ on $\mathbb{R}^{2} \backslash \Omega^{n}$ (the $m$-symmetric extension of $B^{w}$ on $\mathbb{R}^{2} \backslash \Omega^{n}$ ) according with representation $(5.12)$.

Let us introduce the following measures on $\Omega_{\varepsilon}^{n}$

$$
m_{\varepsilon}^{n}(d x)=\mathbf{1}_{\Omega^{n} \cup \Sigma^{n}}(x) d x+\mathbf{1}_{\partial \Omega^{n}}(x) d \mathfrak{s}
$$

and

$$
\mathfrak{m}_{\varepsilon}^{n}(d x)=\mathfrak{m}_{v(n)}(x) d x+2 v(n) \sigma_{n} \mathbf{1}_{\partial \Omega^{n}}(x) d \mathfrak{s}
$$

where the measure on the pre-fractal curve is taken according with Proposition 1. Notice that $\mathfrak{m}_{v}$ is related to $\tilde{\mathfrak{m}}_{v}$ by means of Eqs. 5.3, 5.8, 5.9. Thus, we write (5.6) as $\mathbb{P}_{x}^{n}\left(B_{t}^{v} \in \Lambda\right)$ and

$$
\mathbb{P}_{m_{\varepsilon}^{n}}^{n}\left(B_{t}^{v, *} \in \Lambda\right)=\int_{\Omega^{*}} \mathbb{P}_{x}^{n}\left(B_{t}^{v, *} \in \Lambda\right) m_{\varepsilon}^{n}(d x) .
$$

\subsection{Local Time and Occupation Measure}

The skew BM is a Markov process with continuous paths (and discontinuous local time). The boundary local time is a PCAF defined as an occupation time process on the boundary (see $[21,26]$ for example). Moreover, we deal with a modified skew BM depending on the weights $w^{n}$. For a given $n$, we introduce the occupation density $\ell_{t}^{n}(x), x \in \Omega^{*}, t \geq 0$ such that, for $\Lambda \in \Omega^{*}$, the following occupation formula holds true

$$
\begin{aligned}
\int_{0}^{t \wedge \tau_{\Omega_{\varepsilon}^{n}}} \mathbf{1}_{\Lambda}\left(\widetilde{B_{s}^{v, *}}\right) d s & =\int_{0}^{t} \mathbf{1}_{\Lambda}\left(B_{s}^{v, *}\right) d s \\
& =\int_{\Lambda} \ell_{t}^{n}\left(y ; B^{v, *}\right) m_{\varepsilon}^{n}(d y)=\int_{\Lambda} \ell_{t \wedge \tau_{\Omega_{\varepsilon}^{n}}^{n}}\left(y ; \widetilde{B^{v, *}}\right) m_{\varepsilon}^{n}(d y)
\end{aligned}
$$

where $m_{\varepsilon}^{n}$ is the measure (5.13). With some abuse of notation we do not distinguish here between absolutely continuity of the occupation density on $\Lambda \subset \Omega_{\varepsilon}^{n}$ or $\Lambda \subset \partial \Omega^{n}$. For the sake of simplicity we use the same symbol $\ell_{t}^{n}$ for a density w.r.t. $m_{\varepsilon}^{n}$. In particular, with Eq. 3.4 in mind, as $t \rightarrow 0$ we have that

$$
\begin{aligned}
& \frac{1}{t} \mathbb{E}_{m_{\varepsilon}^{n}}^{n}\left[\int_{0}^{t} f\left(B_{s}^{v, *}\right) d \Gamma_{s}^{\Lambda}\right] \rightarrow \int_{\Omega_{\varepsilon}^{n}} f(x) \mathbf{1}_{\Lambda}(x) d x, \\
& \frac{1}{t} \mathbb{E}_{m_{\varepsilon}^{n}}^{n}\left[\int_{0}^{t} f\left(B_{s}^{v, *}\right) d L_{s}^{\Lambda}\right] \rightarrow \sigma_{n} \int_{\partial \Omega^{n}} f(x) \mathbf{1}_{\Lambda}(x) d \mathfrak{s} .
\end{aligned}
$$


The occupation density $\ell_{t}^{n}\left(y ; B^{\nu, *}\right)$ must be discontinuous on $\partial \Omega^{n}$ (and continuous on $\Omega^{n} \cup \Sigma^{n}$ with different "speed" measures depending on $w^{n}$; recall that $\left.w^{n}\right|_{\Omega^{n}}=1$ ). In particular,

$$
\ell_{t}^{n}\left(y ; B^{v}\right) d y=\ell_{t}^{n}\left(y ; B^{1 / 2}\right) \mathfrak{m}_{v}(d y)
$$

where the process $B^{1 / 2}$ behaves like the $\mathrm{BM}(5.12)$ on $\Omega_{\varepsilon}^{n}$ with $v=1 / 2$ (that is, there is no reflection on $\partial \Omega^{n}$ for $B^{1 / 2}$ ). The occupation density on the boundary can be therefore written by considering the "right" (reflection from the exterior, $\Sigma^{n}$ ) and "left" ( reflection from the interior, $\Omega^{n}$ ) densities. The symmetric local time

$$
\begin{aligned}
\int_{0}^{t} \mathbf{1}_{\partial \Omega^{n}}\left(B_{s}^{v, *}\right) d s & =L_{t}^{\partial \Omega^{n}}\left(B^{v, *}\right) \\
& =\left(L_{t}^{\partial \Omega^{n}-}+L_{t}^{\partial \Omega^{n}+}\right) / 2=\sigma_{n} \int_{\partial \Omega^{n}} \ell_{t}^{n}\left(y ; B^{v, *}\right) m_{\varepsilon}^{n}(d y)
\end{aligned}
$$

is written in terms of $L_{t}^{\partial \Omega^{n}-}=L_{t}^{\partial \Omega^{n}-}\left(B^{\nu, *}\right)$ and $L_{t}^{\partial \Omega^{n}+}=L_{t}^{\partial \Omega^{n}+}\left(B^{\nu, *}\right)$, say "left" and "right" local time. In particular, $\ell_{t}^{n+}\left(y ; B^{\nu, *}\right)=2 v(n) \sigma_{n} \ell_{t}^{n}\left(y ; B^{\nu, *}\right)$ and $\ell_{t}^{n-}\left(y ; B^{v, *}\right)=$ $2(1-v(n)) \sigma_{n} \ell_{t}^{n}\left(y ; B^{\nu, *}\right)$. Recall that we are dealing with the BM $B^{\nu, *}$ such that $B^{\nu, *}=B^{*}$ on $\overline{\Omega^{n}}$ and $B^{\nu, *}=B^{w, *}$ on $\overline{\Sigma^{n}}$ with probability respectively given by $1-v$ and $v$ as in Eq. 5.12. We have that $L_{t}^{\partial \Omega^{n}-}\left(B^{\nu, *}\right)=L_{t}^{\partial \Omega^{n}}\left(B^{*}\right)$ and $L_{t}^{\partial \Omega^{n}+}\left(B^{\nu, *}\right)=L_{t}^{\partial \Omega^{n}}\left(B^{w, *}\right)$ according with Eqs. 5.12 and 5.19, that is

$$
L_{t}^{\partial \Omega^{n}-}\left(B^{v, *}\right)=2(1-v) L_{t}^{\partial \Omega^{n}}\left(B^{\nu, *}\right) \quad \text { and } \quad L_{t}^{\partial \Omega^{n}+}\left(B^{\nu, *}\right)=2 v L_{t}^{\partial \Omega^{n}}\left(B^{v, *}\right)
$$

where $L_{t}^{\partial \Omega^{n}}\left(B^{\nu, *}\right)$ is a symmetric local time (independent from $\left.v\right)$. Since $t \mapsto L_{t}^{\partial \Omega^{n}}\left(B^{\nu, *}\right)$ is a continuous additive functional, formulas in Eq. 5.20 define PCAFs. Indeed, for $\eta>0$, $\eta L \in \mathbf{A}_{c}^{+}$iff $L \in \mathbf{A}_{c}^{+}$([49, Proposition VI.45.10]). The representations (5.20) can be obtained by considering excursions of $\widetilde{B^{v}, *}$ and suitable time changes for example in the case of regular interfaces as in Section 4. According with (5.9), for the sequence of probabilities $v(n)$, it holds that

$$
\frac{1}{v(n)} \frac{c_{n} w^{n}}{1+c_{n} w^{n}} \rightarrow 1 \quad \text { uniformly on } \Sigma^{n} \text { as } n \rightarrow \infty .
$$

Observe that we always have $c_{n} w^{n} \rightarrow 0$ (as $n \rightarrow \infty$ ) as basic assumption between conductivity and thickness of the fiber, the insulating fractal layer case. We use the fact that, for any $f \in \mathcal{B}_{+}$,

$$
\begin{aligned}
\int_{\Lambda} f(y) \ell_{t}^{n+}\left(y ; B^{\nu, *}\right) m_{\varepsilon}^{n}(d y) & =2 v(n) \sigma_{n} \int_{\Lambda} f(y) \ell_{t}^{n}\left(y ; B^{v, *}\right) m_{\varepsilon}^{n}(d y) \\
& =\int_{\Lambda} f(y) \ell_{t}^{n}\left(y ; B^{\nu, *}\right) \mathfrak{m}_{\varepsilon}^{n}(d y), \quad \text { if } \quad \Lambda \subseteq \partial \Omega^{n}(5.22)
\end{aligned}
$$

and

$$
\int_{\Lambda} f(y) \ell_{t}^{n}\left(y ; B^{v, *}\right) m_{\varepsilon}^{n}(d y)=2 v(n) \int_{\Lambda} f(y) \ell_{t}^{n}\left(y ; B^{w}\right) m_{\varepsilon}^{n}(d y), \quad \text { if } \quad \Lambda \subseteq \Sigma^{n}
$$

under $\mathbb{E}_{x}^{n}$. Formulas (5.22) and (5.23) can be also obtained by considering (5.14) together with representation (5.12) and by following similar arguments as in [11]. Indeed, for $0<$ $t_{1}<t_{2}<\tau_{\Omega_{\varepsilon}^{n}}$, and $\Lambda=\operatorname{supp}[\mu]$ where $\mu$ is the Revuz measure of $A_{t}$, we have that

$$
\mathbb{E}_{x}^{n}\left[\int_{t_{1}}^{t_{2}} f\left(B_{s}^{v, *}\right) d A_{s}\right]=\int_{t_{1}}^{t_{2}} d s \int_{\Omega^{*}} f(y) p^{\nu, *}(s, x, y) \mu(d y) .
$$


If $A_{t}=L_{t}^{\Lambda+}$, then

$$
\begin{aligned}
\mathbb{E}_{x}^{n}\left[\int_{0}^{t} f\left(B_{s}^{v, *}\right) d A_{s}\right] & =\int_{0}^{t} d s \int_{\Omega^{*}} f(y) p^{w, *}(s, x, y) \mu(y) \mathfrak{m}_{\varepsilon}^{n}(d y) \\
& =2 v(n) \sigma_{n} \int_{0}^{t} d s \int_{\Lambda} f(y) p^{w, *}(s, x, y) m_{\varepsilon}^{n}(d y)
\end{aligned}
$$

where $p^{w, *}(s, x, y)$ is the transition kernel of $B^{v, *}$ on $\Omega_{\varepsilon}^{n} \backslash \Omega^{n}$ and formula (5.22) follows by Eqs. 5.16 and 5.19. Notice that for $\Lambda \subseteq \Sigma^{n}$ (that is, $A_{t}=\Gamma_{t}^{\Lambda}$ ), the integral (5.23) vanishes as $n \rightarrow \infty$.

For the Neumann heat kernel $p_{N}$ in an inner uniform domain, it holds that ([32])

$$
c_{1} t^{-1} e^{-\frac{d^{2}(x, y)}{c_{2} t}} \leq p_{N}(t, x, y) \leq c_{3} t^{-1} e^{-\frac{d^{2}(x, y)}{c_{4} t}} .
$$

In view of Eq. 5.24 and the Gaussian bound (5.26), there exists $C=C\left(t_{1}, t_{2}\right)>0$ such that

$$
\mathbb{E}_{x}^{n}\left[\int_{t_{1}}^{t_{2}} f\left(B_{s}^{v, *}\right) d A_{s}\right] \leq C \int_{\Omega^{*}} f(y) \mu(d y) .
$$

It is known that the reflecting $\mathrm{BM} B^{+}$spends zero Lebesgue amount of time on the boundary $\partial \Omega$. On the other hand, we are interested in $\Gamma_{t}^{\Omega}$ obtained as a limit of $\Gamma_{t}^{\Omega_{\varepsilon}^{n} \backslash \Omega^{n}}$ for some $t<T$. Moreover, we focus on $L^{\partial \Omega^{n}+}$ and $L^{\partial \Omega^{n}-}$ or equivalently on $\Gamma_{t}^{\Omega_{\varepsilon}^{n} \backslash \Omega^{n}}$ and $\Gamma_{t}^{\Omega_{\varepsilon}^{n} \backslash \Sigma^{n}}$ in our analysis. In order to streamline the notation as much as possible we write $\ell_{t}^{n}$ in place of $\ell_{t}^{n}\left(B^{\nu, *}\right)$ and $\ell_{t}$ instead of $\ell_{t}^{\infty}$ when no confusion arises.

\subsection{The Probabilistic Framework}

Here the aim is to provide a suitable framework to start with in the next section. We formalize some link between the previous sections and Brownian motions on trap domains, in particular on a domain with Koch interfaces. Hereafter, we assume that $d_{n}=0$ and $d=0$ without loss of generality. Moreover, we point out that for the planar BM $B, \forall \Lambda \subseteq \mathbb{R}^{2}$, $\mathbb{P}_{x}$-almost surely, $\int_{0}^{\infty} e^{-\delta s} \mathbf{1}_{\Lambda}\left(B_{s}\right) d s=\infty$ if $\delta=0$. The problem in Theorem 1 can be formulated as follows.

Theorem 6 The unique weak solution of problem (2.10) can be written as

$$
u_{n}(x)=\mathbb{E}_{x}^{n}\left[\int_{0}^{\tau_{\Omega_{\epsilon}^{n}}} e^{-t \delta_{n}} f_{n}\left(\widetilde{B_{t}^{v(n), *}}\right) d t\right]
$$

The associated Dirichlet form on $H_{0}^{1}\left(\Omega_{\varepsilon}^{n}\right)$ is given by Eq. 5.1 or equivalently by Eq. 2.9. The perturbed form $\left(\mathcal{E}_{0}^{\mu_{A^{n}}}, D\left(\mathcal{E}_{0}^{\mu_{A^{n}}}\right)\right)$ is obtained by considering the Revuz measure of the additive functional $A_{t}^{n}$ associated with the killing time $\tau_{\Omega_{\varepsilon}^{n}}$. Let $\infty_{D^{c}}$ be the measure which is $+\infty$ on the complement $D^{c}$ of a Borel set $D$. Formula (3.8) becomes

$$
\mathbf{P}_{t}^{n} f(x)=\mathbb{E}_{x}^{n}\left[f\left(B_{t}^{\nu, *}\right)\right]=\mathbb{E}_{x}^{n}\left[e^{-\overline{A_{t}^{n}}} f\left(\widetilde{B_{t}^{\nu, *}}\right)\right]=\mathbb{E}_{x}^{n}\left[e^{-\delta_{n} t} f\left(\widetilde{B_{t}^{\nu, *}}\right) ; t<\tau_{\Omega_{\varepsilon}^{n}}\right]
$$

where $\overline{A_{t}^{n}}=\delta_{n} t+A_{t}^{n}$ is a PCAF with drift $\delta_{n}\left(\delta_{n} \geq 0\right)$ and associated Revuz measure which can be written as $\mu \overline{A^{n}}(d x)=\delta_{n} d x+\infty_{D^{c}}$ and $D=\Omega_{\varepsilon}^{n}$. The resolvent kernel is written as follows

$$
R_{\lambda}^{n} f(x)=\mathbb{E}_{x}^{n}\left[\int_{0}^{\tau_{\Omega_{\varepsilon}^{n}}} e^{-\lambda t-\delta_{n} t} f\left(\widetilde{B_{t}^{\nu, *}}\right) d t\right]=\mathbb{E}_{x}^{n}\left[\int_{0}^{\infty} e^{-\lambda t-\overline{A_{t}^{n}}} f\left(\widetilde{B_{t}^{\nu, *}}\right) d t\right]
$$


For the sake of simplicity we consider $\delta_{n}=0$ (if not otherwise specified). The case $\delta_{n}>0$ can be immediately obtained by considering $\overline{A_{t}^{n}}$ with $\mu \overline{A^{n}}(d x)=\mu_{A^{n}}(d x)+\delta_{n} d x$ and following similar arguments. We rewrite (3.7) by considering that the semigroup (5.29) generates the Dirichlet form on $L^{2}\left(\Omega_{\varepsilon}^{n}\right)$ given by

$$
\mathcal{E}_{0}^{\mu_{A^{n}}}(u, v)=\tilde{a_{n}}(u, v)+\langle u, v\rangle_{\mu_{A^{n}}}, \quad u, v \in H^{1}\left(\mathbb{R}^{2}\right) \cap L^{2}\left(\mu_{A^{n}}\right)
$$

where

$$
\tilde{a_{n}}(u, v):=(1-v(n)) \int_{\Omega^{n}} \nabla u \nabla v d x+v(n) \int_{\mathbb{R}^{2} \backslash \Omega^{n}} \nabla u \nabla v d x .
$$

Observe that the part process of $\widetilde{B_{t}^{v, *}}$ on $\Omega_{\varepsilon}^{n}$ is transient if and only if $\operatorname{Cap}\left(\mathbb{R}^{2} \backslash \Omega_{\varepsilon}^{n}\right)>0$ ([24, Proposition 3.5.10]). The lifetime is finite and the process is killed. The representation (5.29) says also that for our initial problem (2.10) it can be given a variational formulation as in [16] by considering the measure

$$
\infty_{\left(\Omega_{\varepsilon}^{n}\right)^{c}}(B)=\left\{\begin{array}{ll}
+\infty, & \text { if } \operatorname{Cap}_{1}\left(B \cap\left(\mathbb{R}^{2} \backslash \Omega_{\varepsilon}^{n}\right)\right)>0, \\
0, & \text { otherwise }
\end{array} .\right.
$$

Thus, the Dirichlet condition is prescribed in the capacity sense and the modified BM moves on $\mathbb{R}^{2}$.

We continue with the following representation of the solution in Theorem 2.

Theorem 7 The unique weak solution of Eq. 2.15 can be written as

$$
u(x)=\mathbb{E}_{x}\left[\int_{0}^{\infty} e^{-t \delta_{0}-c_{0} L_{t}^{\partial \Omega}} f\left(B_{t}^{+}\right) d t\right]
$$

where $B^{+}=\left(\left\{B_{t}^{+}\right\}_{t \geq 0} ; \mathfrak{F}^{+} ; \mathbb{P}_{x}, x \in \bar{\Omega}\right)$ is a reflecting $B M$ on $\bar{\Omega}$ and $L_{t}^{\partial \Omega}=L_{t}^{\partial \Omega}\left(B^{+}\right)$is the local time on the boundary $\partial \Omega$.

The associated Dirichlet form, say $\mathcal{E}_{0}^{\mu_{A}}$, is therefore given by Eq. 2.12 with $D\left(\mathcal{E}_{0}^{\mu_{A}}\right)=$ $H^{1}(\Omega) \cap L^{2}\left(c_{0} \mu_{\alpha}\right)$. The solution (5.34) is obtained by considering the exponential random variable $\zeta$ with parameter $c_{0}>0$ (independent from $B^{+}$) and $\zeta^{\Omega}=\inf \left\{s \geq 0: L_{s}^{\partial \Omega} \notin\right.$ $[0, \zeta]\}$. Thus, the associated semigroup is written as

$$
\begin{aligned}
\mathbf{P}_{t}^{+} f(x) & =\mathbb{E}_{x}\left[e^{-\delta_{0} t} f\left(B_{t}^{+}\right) ; t<\zeta^{\Omega}\right]=\mathbb{E}_{x}\left[e^{-\delta_{0} t} f\left(B_{t}^{+}\right) ; \zeta>L_{t}^{\partial \Omega}\right] \\
& =\mathbb{E}_{x}\left[e^{-\delta_{0} t} f\left(B_{t}^{+}\right) \mathbb{E}\left[\zeta>L_{t}^{\partial \Omega} \mid \mathfrak{F}^{+}\right]\right]=\mathbb{E}_{x}\left[f\left(B_{t}^{+}\right) e^{-\delta_{0} t-c_{0} L_{t}^{\partial \Omega}}\right] .
\end{aligned}
$$

Let $A_{t}=\Gamma_{t}^{\Lambda}(B)$ and $A_{t}^{-1}=\inf \left\{s \geq 0: A_{s} \notin[0, t]\right\}$. Since $A_{t}$ is a non-decreasing process, $\left(A_{t}^{-1}<s\right) \equiv\left(A_{s}>t\right)$ and we say that $A^{-1}$ is the inverse of $A$. Obviously we have that $\left(\zeta^{\Omega}>t\right) \equiv\left(L_{t}^{\partial \Omega}<\zeta\right)$. It is worth mentioning that $B_{t}^{+}$can not be written (for all $t>0)$ as $B_{\left(A_{t}\right)^{-1}}$. We can not consider the skew product representation as in Section 4 or in the recent paper [51] for instance. The reflecting BM has been investigated by many researchers and some different constructions have been also considered. Nevertheless, some technical problems can arise from the characterization of the domains. Here we consider a domain with fractal boundary and in particular, we exploit the fact that our pre-fractal and fractal Koch domains are non trap. This permits us to consider occupation measures even if the fractal nature of the boundary does not allow the study of the corresponding time changed processes. Theorem 3 can be formulated as follows. 
Theorem 8 The unique weak solution of problem (2.19) can be written as

$$
u(x)=\mathbb{E}_{x}\left[\int_{0}^{\tau_{\Omega}} e^{-t \delta_{0}} f\left(\widetilde{B}_{t}\right) d t\right]
$$

where $\tau_{\Omega}$ is the first time the $B M \widetilde{B}$ hits the boundary $\partial \Omega$.

The associated Dirichlet form, say $\mathcal{E}_{0}^{\mu_{A}}$, is given by Eq. 2.17 with $D\left(\mathcal{E}_{0}^{\mu_{A}}\right)=$ $H^{1}\left(\mathbb{R}^{2}\right) \cap L^{2}\left(\infty \Omega^{c}\right)$.

We shall approach the convergence in $L^{2}$ of the solutions we are interested in, by first considering convergence of measures. Let $\left\{\mathbb{P}^{n}\right\}_{n}$ be a sequence of probability measures on $(E, \mathfrak{E})$. We say that $\mathbb{P}^{n}$ converges weakly-^ to $\mathbb{P}$ on $(E, \mathfrak{E})$ as $n \rightarrow \infty$ and write $\mathbb{P}^{n} \stackrel{w^{\star}}{\rightarrow} \mathbb{P}$, if $\mathbb{E}^{n} f\left(X^{n}\right)=\int_{E} f d \mathbb{P}^{n} \rightarrow \int_{E} f d \mathbb{P}=\mathbb{E} f(X), \forall f \in C_{b}(E)$ where $X^{n}$ and $X$ are the random variables with probability measures $\mathbb{P}^{n}$ and $\mathbb{P}$ (that is, $X^{n}$ convergences in law to $X$ and we also write $X^{n} \stackrel{\text { law }}{\rightarrow} X$ ). If a sequence of stochastic processes converges (weakly) in the sense of finite-dimensional laws (write $X^{n} \stackrel{f . d .}{\rightarrow} X$ ) we are in need of tightness in order to get convergence in law. Moreover, we write $X^{n} \stackrel{\text { law }}{\rightarrow} \infty$ (meaning also that $X^{n} \stackrel{\text { a.s. }}{\rightarrow} \infty$, that is almost surely or with probability one) if $\forall M, \exists n^{*}: \mathbb{P}\left(X^{n}>M\right)=1, \forall n>n^{*}$. We use vague convergence arguments in this case, that is for a sequence of measures $\mu_{n}$ on $E \cup\{+\infty\}$ we have $\mu_{n} \stackrel{v}{\rightarrow} \mu$ if $\left\langle f, \mu_{n}\right\rangle \rightarrow\langle f, \mu\rangle, \forall f \in C_{0}^{+}$, the class of continuous functions $f: \mathbb{R} \rightarrow \mathbb{R}_{+}$with compact support.

Theorem 9 ([42])The Mosco convergence of the forms is equivalent to the strong convergence of the associated resolvents and semigroups.

Convergence of semigroups, by the Markov property, provides convergence of finite dimensional laws. In particular (let the symbol " $\rightarrow$ " denote strong convergence of semigroups), for the semigroup (5.29), under (2.26) and (2.27), consider that (see Theorems 4 and 5):

i) Robin, under (2.28) with $c_{0}>0$ and $\delta_{0} \geq 0$,

$$
\mathbf{P}_{t}^{n} f_{n}(x) \rightarrow \mathbb{E}_{x}\left[e^{-\delta_{0} t} f\left(B_{t}^{+}\right) ; t<\zeta^{\Omega}\right]
$$

ii) Neumann, under (2.28) with $c_{0}=0$ and $\delta_{0}>0$,

$$
\mathbf{P}_{t}^{n} f_{n}(x) \rightarrow \mathbb{E}_{x}\left[e^{-\delta_{0} t} f\left(B_{t}^{+}\right)\right]
$$

iii) Dirichlet, under (2.31) and $\delta_{0} \geq 0$,

$$
\mathbf{P}_{t}^{n} f_{n}(x) \rightarrow \mathbb{E}_{x}\left[e^{-\delta_{0} t} f\left(\widetilde{B}_{t}\right) ; t<\tau_{\Omega}\right]=\mathbb{E}_{x}\left[e^{-\delta_{0} t} f\left(B_{t}^{+}\right) ; t<\tau_{\Omega}\right] .
$$

Thus, starting from the part process of $\widetilde{B_{t}^{v, *}}$ on $\Omega_{\varepsilon}^{n}$ (and therefore from Eq. 5.29), we simply write

$$
\mathbf{P}_{t}^{n} f_{n}(x) \rightarrow \mathbf{P}_{t} f(x)=\mathbb{E}_{x}\left[e^{-\delta_{0} t} f\left(B_{t}^{+}\right) ; t<T_{c_{\infty}}\right]
$$

where the stopping time depends on $\lim _{n \rightarrow \infty} c_{n}=c_{\infty} \in[0, \infty]$. We arrive at the reflecting BM on $\bar{\Omega}$ stopped by $T_{c_{\infty}}$, that is the lifetime depends on the asymptotic behaviour of the process on the thin layer $\overline{\Sigma^{n}}$. However, the convergence in Eq. 5.39 follows once the 
convergence of a suitable sequence of stopping times to $T_{c_{\infty}}$ in an appropriate sense has been shown. If $m_{n} \rightarrow m$, for the Borel sets $\left\{\Lambda_{j}\right\}$ we have that

$$
\mathbb{E}_{m_{n}}^{n}\left[\mathbf{1}_{\Lambda_{1}}\left(X_{t_{1}}^{n}\right) \cdots \mathbf{1}_{\Lambda_{k}}\left(X_{t_{k}}^{n}\right)\right] \rightarrow \mathbb{E}_{m}\left[\mathbf{1}_{\Lambda_{1}}\left(X_{t_{1}}\right) \cdots \mathbf{1}_{\Lambda_{k}}\left(X_{t_{k}}\right)\right]
$$

as $n \rightarrow \infty$. This is due to the Markov property and the fact that

$$
\mathbb{E}_{x}^{n}\left[\mathbf{1}_{\Lambda_{1}}\left(X_{t_{1}}^{n}\right) \cdots \mathbf{1}_{\Lambda_{k}}\left(X_{t_{k}}^{n}\right)\right]=\mathbf{P}_{t_{1}}^{n} \mathbf{1}_{\Lambda_{1}} \mathbf{P}_{t_{2}-t_{1}}^{n} \mathbf{1}_{\Lambda_{2}} \cdots \mathbf{P}_{t_{k}-t_{k-1}}^{n} \mathbf{1}_{\Lambda_{k}}(x)
$$

where $\mathbf{P}_{t}^{n} f(x)$ is the transition (non conservative) semigroup (5.29). Thus we have conver-

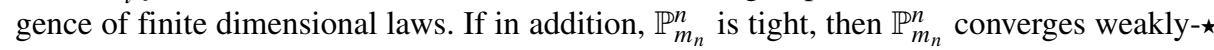
to $\mathbb{P}_{m}$.

Definition 3 The sequence of probability measures $\left\{\mathbb{P}^{n}\right\}_{n}$ on a metric space $E$ is said to be tight if for every $\epsilon>0$, there exists a compact set $K \subseteq E$ such that $\sup _{n} \mathbb{P}^{n}(E \backslash K) \leq \epsilon$.

We use the (Kolmogorov-Chentsov) criterion based on the moments of increments, that is, the sequence $X^{n}$ is tight if $X_{0}^{n}=0$ and there exist $\alpha, \beta>0$ and $C>0$ such that, for $T>0$,

$$
\mathbb{E}\left[\left|X_{t}^{n}-X_{s}^{n}\right|^{\alpha}\right] \leq C|t-s|^{\beta+1}
$$

holds uniformly on $n \in \mathbb{N}$ and $0 \leq s, t \leq T$ (see [36, Corollary 14.9]). Thus, the sequence $X^{n}$ is tight in the space of all continuous processes, equipped with the norm of locally uniform convergence.

\section{Main Results}

We consider occupation measures on both $\Omega_{\varepsilon}^{n}$ and $\partial \Omega^{n}$ (local times) instead of planar Brownian motions. Let $\zeta^{\Omega_{\varepsilon}^{n}}$ be the lifetime of $B_{t}^{\nu, *}$ on $\Omega_{\varepsilon}^{n}$ and $\zeta^{\Omega}$ be the lifetime of the limit process on $\Omega$. Let us focus now on Eq. 5.39. Let $X_{t}^{n}$ be the $m$-version of $B_{t}^{v(n), *}=$ $\left\{\widetilde{B_{t}^{\nu(n), *}}, t<T_{c_{n}}\right\}$ with transition semigroup $\mathbf{P}_{t}^{n}$ (associated with the form $\mathcal{E}_{0}^{\mu_{A^{n}}}$ ) and $X_{t}$ be the process with transition semigroup $\mathbf{P}_{t}$ (associated with the form $\mathcal{E}_{0}^{\mu_{A} \infty}$ ). Our aim is to prove the following theorem.

Theorem 10 Let $A_{t}^{n}$ be the PCAF associated with $M_{t}^{n}=\mathbf{1}_{\left(t<\zeta \Omega_{\varepsilon}^{n}\right)}$ as in Eq. 5.29. We have:

i) $c_{n} \rightarrow c_{0} \in(0, \infty) \Leftrightarrow \zeta^{\Omega_{\varepsilon}^{n}} \stackrel{\text { law }}{\rightarrow} \zeta^{\Omega} \Leftrightarrow \mu_{A^{n}} \stackrel{w}{\rightarrow} \mu_{A^{\infty}}=c_{0} \mu_{\alpha}\left(\mu_{\alpha}\right.$ is defined in Eq. 2.2).

$X_{t}$ is an elastic (or partially reflected) $B M$ on $\bar{\Omega}$;

ii) $\quad c_{n} \rightarrow 0 \Leftrightarrow \zeta^{\Omega_{\varepsilon}^{n}} \stackrel{\text { a.s. }}{\rightarrow} \infty \Leftrightarrow \mu_{A^{n}} \stackrel{w}{\rightarrow} \mu_{A^{\infty}}=0$.

$X_{t}$ is a reflecting $B M$ on $\bar{\Omega}$;

iii) $\quad c_{n} w^{n} \rightarrow 0, c_{n} \rightarrow \infty \Leftrightarrow \zeta^{\Omega_{\varepsilon}^{n}} \stackrel{\text { law }}{\rightarrow} \tau_{\Omega} \Leftrightarrow \mu_{A^{n}} \stackrel{v}{\rightarrow} \mu_{A^{\infty}}=\infty$ (is locally infinite). $X_{t}$ is an absorbing $B M$ on $\Omega$.

The main tools we deal with are stopping times. We first assume that a.s. $\zeta^{\Omega_{\varepsilon}^{n}}=T_{c_{n}} \forall n$, that is the lifetime is equivalent to a random time depending on $c_{n} \geq 0$. Then, we focus on the sequence of random times $T_{c_{n}}$ with $c_{n} \rightarrow c_{\infty} \in[0, \infty]$ as $n \rightarrow \infty$ and we study the convergence $T_{c_{n}} \rightarrow T_{c_{\infty}}$. Thus, $T_{c_{\infty}}$ plays the role of lifetime for the limit BM on $\Omega$ (or $\bar{\Omega}$ ). 
Remark 3 Let $\zeta^{n}$ be a r.v. with density law $\mathbb{P}\left(\zeta^{n} \in d x\right)=c_{n} \exp \left(-c_{n} x\right) \mathbf{1}_{[0, \infty)}(x) d x$. We obviously have that $\mathbb{P}\left(\zeta^{n} \leq x\right)=1-\exp \left(-c_{n} x\right)$ and $\mathbb{E} \zeta^{n}=1 / c_{n}$. We denote by $\zeta^{\infty}$ the limit of $\zeta^{n}$ as $n \rightarrow \infty$ in the following sense.

If $c_{n} \rightarrow \infty$, then $\zeta^{n} \stackrel{\mathbb{P}}{\rightarrow} \zeta^{\infty}=0$. Indeed, by Markov's inequality, we have that $\mathbb{P}\left(\mid \zeta^{n}-\right.$ $\left.\zeta^{\infty} \mid>\epsilon\right) \leq \epsilon^{-1} \mathbb{E}\left|\zeta^{n}\right| \rightarrow 0$ as $c_{n} \rightarrow \infty$. Moreover, $\zeta^{\infty} \geq 0$ with $\mathbb{E} \zeta^{\infty}=0$. Thus, $\mathbb{P}\left(\zeta^{\infty}=0\right)=1$.

If $c_{n} \rightarrow 0$ we have that $\mathbb{P}\left(\zeta^{\infty} \leq x\right)=0$ for all $x \in[0, \infty)$ with $\zeta^{\infty} \geq 0$ and, by observing that $\mathbb{E} \zeta^{\infty}=\infty$, we conclude that $\mathbb{P}\left(\lim _{n \rightarrow \infty} \zeta^{n}=\zeta^{\infty}=\infty\right)=1$.

If $c_{n} \rightarrow c_{0} \in(0, \infty)$, we simply have that $\zeta^{n} \stackrel{\operatorname{law}}{\rightarrow} \zeta^{\infty}=\zeta$ as $n \rightarrow \infty$ where $\zeta$ is the exponential r.v. with parameter $c_{0}$.

We can also relate a r.v. $\zeta^{n}$ to the time the process $B_{t}^{v, *}$ spends on (or cross) the pre-fractal $\partial \Omega^{n}$ as follows. For a fixed $n$, denote by $\widehat{\zeta^{\Omega^{n}}}$ the r.v. written as

$$
\widehat{\zeta^{\Omega^{n}}}:=\inf \left\{0<s \leq \zeta^{\Omega_{\varepsilon}^{n}}: L_{s}^{\partial \Omega^{n}}\left(\widetilde{B^{\nu, *}}\right)>\zeta^{n}\right\}=\inf \left\{s>0: L_{s}^{\partial \Omega^{n}}\left(B^{\nu, *}\right)>\zeta^{n}\right\}
$$

assuming that $\zeta^{n}$ is independent from $B^{\nu, *}$ (and therefore, from the local time on the prefractal boundary). Obviously, $\zeta^{\Omega^{n}}$ is a sequence of Markov stopping times before absorption on $\partial \Omega_{\varepsilon}^{n}$. To be clear, $\zeta^{\Omega^{n}} \leq \zeta^{\Omega_{\varepsilon}^{n}}$ with probability one, $\forall n \in \mathbb{N}$ and Eq. 6.1 can be rewritten as

$$
\widehat{\zeta^{n}}=\tau_{\Omega_{\varepsilon}^{n}} \wedge \widehat{T_{c_{n}}}
$$

in terms of the first exit time $\tau_{\Omega_{\varepsilon}^{n}}$ and $\widehat{T_{c_{n}}}:=\inf \left\{s>0: L_{s}^{\partial \Omega^{n}}\left(\widetilde{B_{s}^{v, *}}\right)>\zeta^{n}\right\}$ with $\zeta^{n}$ exponentially distributed, that is $\widehat{T_{c_{n}}}$ corresponds to the elastic boundary condition. We also observe that (6.1) can be regarded as the lifetime of the process up to the last visit on $\overline{\Omega^{n}}$ assuming that $\zeta^{n}$ is the time the process spends on the pre-fractal boundary before absorption on $\partial \Omega_{\varepsilon}^{n}$. In this case we have the lifetime on $\Omega^{n}$ written as (see also Remark 1)

$$
\zeta^{\Omega^{n}}=\sup \left\{s>0: B_{s}^{\nu(n), *} \in \overline{\Omega^{n}}\right\}=\sigma_{\overline{\Omega^{n}}}
$$

which is no longer Markovian. Moreover, $\mathbb{P}_{x}\left(\widehat{\zeta^{\Omega_{\varepsilon}^{n}}}>\zeta^{\Omega^{n}}\right)>0, m$-a.e. $x$ and

$$
\mathbb{P}_{x}\left(\sigma_{\overline{\Omega^{n}}}>0\right)=\mathbb{P}_{x}\left(\tau_{\Omega^{n}}<\infty\right) .
$$

We consider (6.1) with exponential threshold $\zeta^{n}$ given in Remark 3.

Remark 4 From the discussion in the previous remark, we have the following cases.

If $c_{n} \rightarrow \infty$, then $\zeta^{n} \stackrel{\mathbb{P}}{\rightarrow} 0$ and

$$
\widehat{\zeta^{\Omega^{n}}} \stackrel{\text { law }}{\rightarrow} \inf \left\{s>0: L_{s}^{\partial \Omega}\left(B^{+}\right)>0\right\}=: \tau_{\Omega}
$$

provided that the occupation time sequence $L_{t}^{\partial \Omega^{n}}$ converges in law to the local time $L_{t}^{\partial \Omega}$ of the corresponding limit process.

Similarly, as $c_{n} \rightarrow 0, \mathbb{P}\left(\lim _{n \rightarrow \infty} \zeta^{n}=\infty\right)=1$ and then

$$
\widehat{\zeta^{\Omega^{n}}} \stackrel{\text { law }}{\rightarrow} \inf \left\{s>0: L_{s}^{\partial \Omega}\left(B^{+}\right)=\infty\right\}=\infty .
$$

If $c_{n} \rightarrow c_{0} \in(0, \infty)$, then $\zeta^{n} \stackrel{\text { law }}{\rightarrow} \zeta$ and the lifetime of the limit process depends on the random variable $\zeta$ with parameter $c_{0}$. In particular, we have that

$$
\widehat{\zeta^{\Omega^{n}}} \stackrel{\text { law }}{\rightarrow} \zeta^{\Omega}=\inf \left\{s>0: L_{s}^{\partial \Omega}\left(B^{+}\right)>\zeta\right\}
$$

provided the convergence in law of the occupation time process on the fractal boundary $\partial \Omega$. 
For the sake of simplicity we write $\zeta^{\Omega}$ in place of $\zeta^{\Omega^{\infty}}$. We focus on the PCAF

$$
\widetilde{A_{t}^{n+}}=\Gamma_{t}^{\Sigma^{n}}\left(\widetilde{B^{v, *}}\right)+L_{t}^{\partial \Omega^{n}+}\left(\widetilde{B^{v, *}}\right)=\Gamma_{t}^{\Omega_{\varepsilon}^{n} \backslash \Omega^{n}}\left(\widetilde{B^{v, *}}\right)
$$

and $\int_{0}^{t \wedge \tau_{\Omega_{\varepsilon}^{n}}} d \widetilde{A_{s}^{n+}}$ is the occupation time process on $\Omega_{\varepsilon}^{n} \backslash \Omega^{n}$ of the skew BM $B^{v, *}$. Let us write

$$
R_{\lambda}^{n} \mu_{n}^{+}(x)=2\left(1+c_{n}\right)^{-1} c_{n} \sigma_{n} \mathbb{E}_{x}^{n}\left[\int_{0}^{\zeta^{\Omega_{\varepsilon}^{n}}} e^{-\lambda t-t \delta_{n}} \mathbf{1}_{\partial \Omega^{n}}\left(\widetilde{B_{t}^{\nu(n), *}}\right) d t\right]
$$

where $\mu_{n}^{+}(d x)=2\left(1+c_{n}\right)^{-1} c_{n} \sigma_{n} \mathbf{1}_{\partial \Omega^{n}}(x) m_{\varepsilon}^{n}(d x)$. Let $A_{t}^{n+} \in \mathbf{A}_{c}^{+}$be in Revuz correspondence with the measure $\mu_{n}^{+}$.

Let us consider the symmetric local time

$$
L_{t}^{\partial \Omega}=\left(L_{t}^{\partial \Omega+}+L_{t}^{\partial \Omega-}\right) / 2
$$

as in Eq. 5.19.

Proposition 2 Under (2.14), the boundary local time $\left\{L_{t}^{\partial \Omega+}, t<\zeta^{\partial \Omega}\right\}$ is the unique PCAF such that, for any $x \in \Omega_{\varepsilon}^{n}$,

$$
R_{\lambda}^{n} \mu_{n}^{+}(x) \rightarrow \mathbb{E}_{x}\left[\int_{0}^{\zeta^{\Omega}} e^{-\lambda t-\delta_{0} t} d L_{t}^{\partial \Omega+}\right] \quad \text { as } n \rightarrow \infty .
$$

Proof First we notice that $\mu_{n}$ has finite energy integral. Indeed,

$$
\int_{\Omega^{*}} v(x) \mu_{n}^{+}(d x) \leq\left(\mu_{n}^{+}\left(\partial \Omega^{n}\right)\right)^{1 / 2}\|v\|_{L^{2}\left(\partial \Omega^{n}\right)}=2\left(1+c_{n}\right)^{-1} c_{n} \sqrt{\sigma_{n}}\|v\|_{L^{2}\left(\partial \Omega^{n}\right)} .
$$

From [20, Theorem 8.1] we know that

$$
\|v\|_{L^{2}\left(\partial \Omega^{n}\right)}^{2} \leq \frac{C}{\sigma_{n}}\|v\|_{H^{1}\left(\mathbb{R}^{2}\right)}^{2}
$$

where $C$ is independent of $n$. Since $\left(1+c_{n}\right)^{-1} c_{n} \leq 1$, by extension theorem (see [20, Theorem A.3]) we obtain that $\left\langle v, \mu_{n}^{+}\right\rangle \leq \sqrt{C}\|v\|_{H^{1}\left(\Omega_{\varepsilon}^{n}\right)}$ and $\mu_{n}^{+} \in S_{0}$. Since, under (2.14), $R_{\lambda}^{n} \mu_{n}^{+}(x)$ is bounded (and in view of [24, Lemma 4.1.5]) we have that $\mu_{n}^{+} \in S_{00}$. Let $c_{\infty} \in[0, \infty]$ be such that $c_{n} \rightarrow c_{\infty}$. From Eq. 2.30, for all $f \in \mathcal{B}_{+}$we get that

$$
\left\langle f, \mu_{n}^{+}\right\rangle \rightarrow 2\left(1+c_{\infty}\right)^{-1} c_{\infty} \int_{\partial \Omega} f d \mu \quad \text { as } n \rightarrow \infty .
$$

For a fixed $n$, consider the occupation measure (6.6). In view of Eqs. 5.16, 5.19, 5.22 and 5.23, we write

$$
\begin{aligned}
& \mathbb{E}_{x}^{n}\left[\int_{0}^{t \wedge \tau_{\Omega_{\varepsilon}^{n}}} f\left(\widetilde{B_{s}^{v(n), *}}\right) d \widetilde{A_{s}^{n+}}\right] \\
= & 2 v(n) \mathbb{E}_{x}^{n}\left[\int_{0}^{t \wedge \tau_{\Omega_{\varepsilon}^{n}}} f\left(\widetilde{B_{s}^{\nu(n), *}}\right) d \Gamma_{s}^{\Sigma^{n}}+\int_{0}^{t \wedge \tau_{\Omega_{\varepsilon}^{n}}} f\left(\widetilde{B_{s}^{\nu(n), *}}\right) d L_{s}^{\partial \Omega^{n}}\right]
\end{aligned}
$$

where $\Gamma_{t}^{\Sigma^{n}}=\Gamma_{t}^{\Sigma^{n}}\left(B^{w}\right)$. Set $U_{n}^{\lambda} f(x)=\mathbb{E}_{x}^{n}\left[\int_{0}^{\infty} e^{-\lambda t-\delta_{n} t} f\left(\widetilde{B_{t}^{v, *}}\right) \mathbf{1}_{\left(t<\zeta^{\left.\Omega_{\varepsilon}^{n}\right)}\right.} d \widetilde{A_{t}^{n+}}\right]$. From Eq. 5.17 and the fact that $\mathbb{E}_{x}^{n}\left[\mathbf{1}_{(0<\zeta} \Omega_{\varepsilon}^{n}\right)=\mathbb{P}_{x}^{n}\left(\zeta^{\Omega_{\varepsilon}^{n}}>0\right)=1$ for all $x \in \Omega_{\varepsilon}^{n}$ we obtain

$$
\lim _{\lambda \rightarrow \infty}\left\langle\lambda U_{n}^{\lambda} f, m_{\varepsilon}^{n}\right\rangle=\left\langle f,{\widetilde{\mu_{n}}}^{+}\right\rangle, \quad \forall f \in \mathcal{B}_{+}
$$


where ${\widetilde{\mu_{n}}}^{+}(d x)=2 v(n)\left(\mathbf{1}_{\Sigma^{n}}+\sigma_{n} \mathbf{1}_{\partial \Omega^{n}}\right) m_{\varepsilon}^{n}(d x)$ on $\Omega_{\varepsilon}^{n} \backslash \Omega^{n}$ and therefore, by [30, Theorem 5.1.4] there exists a unique PCAF of $B_{t}^{v, *}$ in Revuz correspondence with ${\widetilde{\mu_{n}}}^{+}$, that is Eq. 6.6. Notice also that, since $v(n) \leq 1$, we can follow the same arguments as before in order to see that ${\widetilde{\mu_{n}}}^{+} \in S_{0}$. Let us consider $\xi_{n} \in \Sigma^{n}$ and $0 \leq M_{n}<\infty$. Since condition (5.21) holds true, we get that

$$
\lim _{n \rightarrow \infty}\left\langle f,{\widetilde{\mu_{n}}}^{+}\right\rangle=\lim _{n \rightarrow \infty}\left(2 M_{n} \frac{c_{n} w^{n}\left(\xi_{n}\right)}{1+c_{n} w^{n}\left(\xi_{n}\right)} f\left(\xi_{n}\right)+2 \frac{c_{n}}{1+c_{n}} \sigma_{n} \int_{\partial \Omega^{n}} f d \mathfrak{s}\right)
$$

and therefore, from Proposition 1 and Eq. 2.32,

$$
\left\langle f,{\widetilde{\mu_{n}}}^{+}\right\rangle \rightarrow 2\left(1+c_{\infty}\right)^{-1} c_{\infty} \int_{\partial \Omega} f d \mu, \quad \text { as } n \rightarrow \infty
$$

where $\mu=\mu_{\alpha}$ is the only Borel measure which is in Revuz correspondence with the "symmetric" local time $L_{t}^{\partial \Omega}$. Indeed, according with Eq. 3.4 and the setting in Eq. 3.7 with $E=\bar{\Omega}$, we have that

$$
\lim _{\lambda \rightarrow \infty} \lambda \mathbb{E}_{m}\left[\int_{0}^{\infty} e^{-\lambda t} f\left(B_{t}\right) d L_{t}^{\partial \Omega}\right]=\int_{\partial \Omega} f d \mu .
$$

Notice that

$$
R_{\lambda}^{n} \mu_{n}^{+}(x) \rightarrow 2\left(1+c_{\infty}\right)^{-1} c_{\infty} \mathbb{E}_{x}\left[\int_{0}^{\zeta^{\Omega}} e^{-\lambda t-\delta_{0} t} \mathbf{1}_{\partial \Omega}\left(B_{t}^{+}\right) d t\right]<\infty
$$

according with Eq. 5.39. From the one to one correspondence between (6.6) and its Revuz measure $\widetilde{\mu_{n}}+$ we prove (6.7) and the claim.

We also focus on the PCAF

$$
\widetilde{A_{t}^{n-}}=\Gamma_{t}^{\Omega^{n} \backslash D^{n}}\left(\widetilde{B^{v, *}}\right)+L_{t}^{\partial \Omega^{n}-}\left(\widetilde{B^{\nu, *}}\right)=\Gamma_{t}^{\Lambda_{n}}\left(\widetilde{B^{v, *}}\right)
$$

where $D_{j}^{n}$ is an increasing sequence of open sets such that $D^{n}=\cup_{j} D_{j}^{n} \rightarrow \Omega$ and $\Lambda_{n}=$ $\overline{\Omega^{n}} \backslash D_{n}$. Let us write

$$
R_{\lambda}^{n} \mu_{n}^{-}(x)=2\left(1+c_{n}\right)^{-1} \sigma_{n} \mathbb{E}_{x}^{n}\left[\int_{0}^{\zeta^{\Omega_{\varepsilon}^{n}}} e^{-\lambda t-t \delta_{n}} \mathbf{1}_{\partial \Omega^{n}}\left(\widetilde{B_{t}^{\nu(n), *}}\right) d t\right]
$$

where $\mu_{n}^{-}(d x)=2\left(1+c_{n}\right)^{-1} \sigma_{n} \mathbf{1}_{\partial \Omega^{n}}(x) m_{\varepsilon}^{n}(d x)$. Let $A_{t}^{n-} \in \mathbf{A}_{c}^{+}$be in Revuz correspondence with the measure $\mu_{n}^{-}$.

Proposition 3 Under (2.14), the boundary local time $\left\{L_{t}^{\partial \Omega-}, t<\zeta^{\Omega}\right\}$ is the unique PCAF such that, for any $x \in \Omega_{\varepsilon}^{n}$,

$$
R_{\lambda}^{n} \mu_{n}^{-}(x) \rightarrow \mathbb{E}_{x}\left[\int_{0}^{\zeta^{\Omega}} e^{-\lambda t-\delta_{0} t} d L_{t}^{\partial \Omega-}\right], \quad \text { as } n \rightarrow \infty
$$

Proof We basically follows the proof of Proposition 2. Indeed, we can write the left local time by considering (6.9) instead of Eq. 6.6 and the density $\ell_{t}^{n-}(y)=2(1-v(n)) \sigma_{n} \ell_{t}^{n}(y)$ as indicated in Section 5.1. We get that ${\widetilde{\mu_{n}}}^{-}(d x)=2(1-v(n)) \sigma_{n} m_{\varepsilon}^{n}(d x)$ is the Revuz measure of $\widetilde{A_{t}^{n-}}$. The result follows from the previous proof of Proposition 2. 
From the previous results we have that $\mu_{n}^{+}$is the Revuz measure of

$$
A_{t}^{n+}=2 \frac{c_{n}}{1+c_{n}} \sigma_{n} \int_{\partial \Omega^{n}} \ell_{t}^{n}(y) m_{\varepsilon}^{n}(d y)=2 \frac{c_{n}}{1+c_{n}} L_{t}^{\partial \Omega^{n}}
$$

and $\mu_{n}^{-}$is the Revuz measure of

$$
A_{t}^{n-}=2 \frac{1}{1+c_{n}} \sigma_{n} \int_{\partial \Omega^{n}} \ell_{t}^{n}(y) m_{\varepsilon}^{n}(d y)=2 \frac{1}{1+c_{n}} L_{t}^{\partial \Omega^{n}} .
$$

As we can immediately see $\left(A_{t}^{n+}+A_{t}^{n-}\right) / 2=L_{t}^{\partial \Omega^{n}}$. Nevertheless, $A_{t}^{n+}$ and $A_{t}^{n-}$ behave respectively like $L_{t}^{\partial \Omega^{n}+}$ and $L_{t}^{\partial \Omega^{n}-}$ only as $n \rightarrow \infty$. As we observed in Section 5.1, the local time $L_{t}^{\partial \Omega^{n}}$ of the process $B_{t}^{\nu, *}$ is given by

$$
L_{t}^{\partial \Omega^{n}}=\sigma_{n} \int_{\partial \Omega^{n}} \ell_{t}^{n}(y) m_{\varepsilon}^{n}(d y) .
$$

According with Proposition 1, we now study the convergence of Eq. 6.11 to

$$
L_{t}^{\partial \Omega}=\int_{\partial \Omega} \ell_{t}(y) d \mu
$$

with $L_{t}^{\partial \Omega} \in \mathbf{A}_{c}^{+}$(see for example [8] for the existence and other properties of Eq. 6.12). The connection between tightness on the line and continuity of the limit process has been pointed out starting from [2,3]. The convergence in law of Eq. 6.11 is proved in the following Proposition 4 and Proposition 5. We begin with the following result concerning the tightness of the sequence $L_{t}^{\partial \Omega^{n}}, t<T$.

Proposition 4 The sequence $\left\{L_{t}^{\partial \Omega^{n}}\right\}_{n}$ is tight in $C([0, T],[0, \infty))$.

Proof Let us consider the sets $\Lambda_{n} \subseteq \partial \Omega^{n}$. The process $L_{t}^{\Lambda_{n}}, t<T$ is a continuous additive functional of zero energy ([24, pag. 149]) and $\mathbb{E}_{x}\left[\left|L_{t}^{\Lambda_{n}}\right|, t<T\right]<\infty$ q.e. $x$. Indeed, from Eqs. 5.20 and 5.27, we have that

$$
\begin{aligned}
\mathbb{E}_{x}^{n}\left[\int_{s}^{t} d L_{u}^{\Lambda_{n}-}\right]+\mathbb{E}_{x}^{n}\left[\int_{s}^{t} d L_{u}^{\Lambda_{n}+}\right] & =2 \mathbb{E}_{x}^{n}\left[\int_{s}^{t} d L_{u}^{\Lambda_{n}}\right] \\
& \leq \text { const } \cdot(t-s) \cdot \sigma_{n} \int_{\Lambda_{n}} m_{\varepsilon}^{n}(d y) .
\end{aligned}
$$

We have that $L_{0}^{\Lambda_{n}}=0$ for all $n$ and, for $k>1$ and $c_{1}>0$,

$$
\mathbb{E}_{x}\left[\left|L_{t}^{\Lambda_{n}}-L_{s}^{\Lambda_{n}}\right|^{k}\right] \leq \sigma_{n} m_{\varepsilon}^{n}\left(\Lambda_{n}\right) c_{1}|t-s|^{k}<c_{1}|t-s|^{k} .
$$

Indeed, for $s<t$,

$$
\begin{aligned}
\mathbb{E}_{x}\left[\int_{s}^{t} d L_{z}^{\Lambda_{n}}\right]^{k} & =k ! \int_{s}^{z_{1}} \ldots \int_{z_{k-1}}^{t} \mathbb{E}_{x}\left[\mathbf{1}_{\Lambda_{n}}\left(B_{z_{1}}^{\nu(n), *}\right) \ldots \mathbf{1}_{\Lambda_{n}}\left(B_{z_{k}}^{v(n), *}\right)\right] d z_{1} \ldots d z_{k} \\
& \leq k ! \int_{s}^{t} \ldots \int_{s}^{t} \mathbb{E}_{x}\left[\mathbf{1}_{\Lambda_{n}}\left(B_{z_{1}}^{\nu(n), *}\right) \ldots \mathbf{1}_{\Lambda_{n}}\left(B_{z_{k}}^{\nu(n), *}\right)\right] d z_{1} \ldots d z_{k}
\end{aligned}
$$

We recall (5.26) and the fact that $\mathbf{P}_{t}^{D} \leq \mathbf{P}_{t}^{N}$ a.e., the transition function of the reflecting BM dominates that of the absorbing BM. From Eqs. 5.41 and 6.13 we can write (6.14). Since $k>1$, a Kolmogorov-type criterion shows the tightness. 
Since $\widehat{\zeta^{\Omega^{n}}} \leq \zeta^{\Omega_{\varepsilon}^{n}}$ we can write $\mathbb{P}_{x}\left(\zeta^{\Omega_{\varepsilon}^{n}} \leq t\right) \leq \mathbb{P}_{x}\left(\widehat{\zeta \Omega^{n}} \leq t\right)=\mathbb{P}_{x}\left(\zeta^{n} \leq L_{t}^{\partial \Omega^{n}}\right)=$ $1-\mathbb{E}_{x} e^{-c_{n} L_{t}^{\partial \Omega^{n}}}$ and, equivalently $\mathbb{P}_{x}\left(\zeta^{\Omega_{\varepsilon}^{n}}>t\right) \geq \mathbb{E}_{x} e^{-c_{n} L_{t}^{\partial \Omega^{n}}}$. Assume that $u$ is the bounded solution to $\partial_{t} u=\mathcal{L}_{n} u-\widehat{\kappa_{n}}(x) u$ with $u_{0}=1$ in $H^{1}\left(\mathbb{R}^{2}\right) \cap L^{2}\left(\mathbb{R}^{2}, \sigma_{n} \mathbf{1}_{\partial \Omega^{n}} d \mathfrak{s}\right)$ where $\widehat{\kappa_{n}}=c_{n} \mathbf{1}_{\partial \Omega^{n}}$. Then, as $t \rightarrow 0, t^{-1}(1-u(x, t)) \rightarrow-\mathcal{L}_{n} u_{0}+\widehat{\kappa_{n}}(x) u_{0}=\widehat{\kappa_{n}}(x)$ gives the killing rate which can be also obtained as ([12])

$$
\lim _{t \rightarrow 0} t^{-1} \mathbb{P}_{x}\left(B_{t}^{\nu, *} \text { is killled in the time interval }(0, t]\right) .
$$
that

Since for $A_{t}=\int_{0}^{t} f\left(X_{s}\right) d s, d e^{A_{t}} / d t=f\left(X_{t}\right) e^{A_{t}}$ and $e^{A_{t}}-1=\int_{0}^{t} f\left(X_{s}\right) e^{A_{s}} d s$, we get

$$
\lim _{t \rightarrow 0} t^{-1}\left(1-\mathbb{E}_{x} e^{-c_{n} L_{t}^{\partial \Omega^{n}}}\right)=\lim _{t \rightarrow 0} \mathbb{E}_{x}\left[c_{n} \mathbf{1}_{\partial \Omega^{n}}\left(B_{t}^{\nu, *}\right)\right]=\widehat{\kappa_{n}}(x) .
$$

Then, $u(x, t)=\mathbb{P}_{x}^{n}\left(\widehat{\zeta^{\Omega^{n}}}>t\right)$. We use the symbol $\widehat{\kappa_{n}}$ in order to underline the connection with the transition semigroup

$$
\left.\widehat{\mathbf{P}_{t}^{n}} f(x):=\mathbb{E}_{x}^{n}\left[f\left(\widehat{\left(B_{t}^{v(n), *}\right.}\right) ; t<\widehat{\zeta^{\Omega^{n}}}\right]\right)=\mathbb{E}_{x}^{n}\left[e^{-\widehat{A_{t}^{n}}} f\left(B_{t}^{\nu(n), *}\right)\right]
$$

with resolvent $\widehat{R_{\lambda}^{n}}$ where $\widehat{\zeta^{\Omega^{n}}}$ is defined as in Eq. 6.1. For $\delta_{n}$ identically zero,

$$
\widehat{\mathbf{P}_{t}^{n}} \mathbf{1}_{\Omega_{\varepsilon}^{n}}(x)=\mathbb{E}_{x}\left[\mathbf{1}_{\Omega_{\varepsilon}^{n}}\left(\widehat{B_{t}^{v(n), *}}\right) ; t<\widehat{\zeta^{\Omega^{n}}}\right]=\mathbb{P}_{x}^{n}\left(\widehat{\zeta^{\Omega^{n}}}>t\right) .
$$

If $\delta_{n}>0$ we have that $\widehat{\mathbf{P}_{t}^{n}} \mathbf{1}_{\Omega_{\varepsilon}^{n}}(x)=e^{-\delta_{n} t} \mathbb{P}_{x}^{n}\left(\widehat{\zeta^{\Omega^{n}}}>t\right)$.

The heat equation solution with Robin boundary conditions has been studied using a Feynamn-Kac formula and a theorem of Ray and Knight on Brownian local time in [8]. In [28], the authors reviewed Kac's method by underlining the connection between the higherorder moments of $A_{\kappa}=\int_{0}^{T} \kappa\left(X_{t}\right) d t$ and the Feynman-Kac formula. Thus, $\mathbb{E}_{m}\left[\left(A_{\kappa}\right)^{k}\right]=$ $k ! m G_{\kappa}^{k} \mathbf{1}$ where $G_{\kappa} w(x)=\int G(x, d y) \kappa(y) w(y)$ (here $m$ is an arbitrary initial distribution). In particular,

$$
f(x)=\mathbb{E}_{x}\left[\exp \int_{0}^{T} \kappa\left(B_{t}\right) d t\right]=\sum_{k=0}^{\infty} G_{\kappa}^{k} \mathbf{1}(x), \quad x \in \Lambda
$$

is finite if and only if is the minimal solution to $f(x)=1+\int \kappa(y) f(y) G(x, d y)$ where $\kappa: \Lambda \mapsto[0, \infty)$ is a measurable Borel function, $T=\tau_{\Lambda}$ and $G$ is the Green function of the killed BM on the boundary of the open set $\Lambda$.

Proposition 5 For $x \in \Omega_{\varepsilon}^{1}, \forall t \geq 0$,

$$
\int_{0}^{\infty} e^{-c_{n} l} \mathbb{P}_{x}^{n}\left(L_{t}^{\partial \Omega^{n}} \in d l\right) \rightarrow \int_{0}^{\infty} e^{-c_{\infty} l} \mathbb{P}_{x}\left(L_{t}^{\partial \Omega} \in d l\right), \quad \text { as } n \rightarrow \infty
$$

$m$-a.e. $x$, for every sequence $c_{n} \geq 0$ such that $c_{n} \rightarrow c_{\infty} \in[0, \infty]$.

Proof The higher-order moment of the boundary local time can be written as in formula (6.15) with $s=0$. Then, by applying (5.40) we have that $\mathbb{E}_{x}^{n}\left[\left(L_{t}^{\partial \Omega^{n}}\right)^{k}\right] \rightarrow \mathbb{E}_{x}\left[\left(L_{t}^{\partial \Omega}\right)^{k}\right]$ weakly (continuously on $t \geq 0, m$-a.e. $x$ ). Indeed, we have weak convergence of finitedimensional distributions from Theorem 9 and tightness from Proposition 4.

Consider the expansion (6.18). We can also write $v_{\widehat{\kappa_{n}}}(x, t)=\mathbb{E}_{x}^{n} \exp -c_{n} L_{t}^{\partial \Omega^{n}}$ for $t \leq$ $T_{c_{n}}$ in terms of Eq. 6.15 with $s=0$. For $c_{n}=\lambda \geq 0 \forall n, v_{\widehat{\kappa_{n}}}$ is the Laplace transform of $\mathbb{P}_{x}^{n}\left(L_{t}^{\partial \Omega^{n}} \in d l\right) / d l$. Since, $\mathbb{P}_{x}^{n} \stackrel{w^{\star}}{\rightarrow} \mathbb{P}_{x}, m$-a.e. $x$ (from the convergence of moments), then the convergence holds for every $c_{n} \geq 0$ (and therefore, for every $c_{\infty} \in[0, \infty]$ ). 
We can write $A_{t}^{n}=\int_{0}^{t} \kappa_{n}\left(\widetilde{B_{s}^{v(n), *}}\right) d s$ in Eq. 5.29 where $\kappa_{n}(\cdot)$ is the state dependent rate for the Markov killing time $T_{C_{n}}$. Thus, we can focus on the multiplicative functional $M_{t}^{n}=e^{-A_{t}^{n}}$ associated with the stopping time $T_{c_{n}}$ and therefore, with the sub-Markov semigroup (5.39). In particular, (5.39) characterizes $M_{t}^{n}$ uniquely ([12, Proposition 1.9]). Also we write $\overline{M_{t}^{n}}=e^{-\overline{A_{t}^{n}}}$ (see formula (5.29)). Notice that (for $\lambda \geq 0$ )

$$
\begin{aligned}
& R_{\lambda}^{n} f(x)=\mathbb{E}_{x}^{n}\left[\int_{0}^{\infty} e^{-\lambda t} f\left(\widetilde{B_{t}^{\nu, *}}\right) \overline{M_{t}^{n}} d t\right] \\
& \widehat{R_{\lambda}^{n}} f(x)=\mathbb{E}_{x}^{n}\left[\int_{0}^{\tau_{\Omega_{\varepsilon}^{n}}} e^{-\lambda t-\delta_{n} t} f\left(\widetilde{B_{t}^{\nu, *}}\right) \widehat{M_{t}^{n}} d t\right]
\end{aligned}
$$

where $\widehat{M_{t}^{n}}=\exp \left(-\widehat{A_{t}^{n}}\right)$ with $\delta_{n}, c_{n}$ as in Eq. 2.14 and $\widehat{A_{t}^{n}}=\int_{0}^{t} \widehat{\kappa_{n}}\left(\widehat{B_{s}^{v(n), *}}\right) d s=c_{n} L_{t}^{\partial \Omega^{n}}$.

The fundamental principle in our investigation is to approximate $M$ by $\widehat{M}$ and transfer the properties known for the approximating functional.

Theorem $11 \widehat{\zeta^{\Omega^{n}}} \stackrel{\text { law }}{\rightarrow} T_{c_{\infty}}$ and under (2.26), $\widehat{R_{\lambda}^{n}} f_{n} \rightarrow R_{\lambda} f$ strongly in $L^{2}(\Omega)$.

Proof First we show that $\widehat{\zeta^{n}} \stackrel{\text { law }}{\rightarrow} T_{c_{\infty}}$ as $c_{n} \rightarrow c_{\infty}$. Let us write $\zeta^{n}=\varsigma / c_{n}$ with $\mathbb{P}(\varsigma>$ $x)=e^{-x}, x \geq 0$. Thus, a.s. $\zeta^{n} \rightarrow \zeta^{\infty}$. Assume $\delta_{n}=0$ and $\lambda>0$. From $\widehat{\mathbf{P}_{t}^{n}}$ we write the associated resolvent

$$
\widehat{R_{\lambda}^{n}} \mathbf{1}_{\Omega_{\varepsilon}^{n}}(x)=\int_{0}^{\infty} e^{-\lambda t} \widehat{\mathbf{P}_{t}^{n}} \mathbf{1}_{\Omega_{\varepsilon}^{n}}(x) d t=\int_{0}^{\infty} e^{-\lambda t} \mathbb{P}_{x}^{n}\left(\widehat{\zeta^{\Omega^{n}}}>t\right) d t
$$

and, from the resolvent (5.30),

$$
R_{\lambda}^{n} \mathbf{1}_{\Omega_{\varepsilon}^{n}}(x)=\int_{0}^{\infty} e^{-\lambda t} \mathbf{P}_{t}^{n} \mathbf{1}_{\Omega_{\varepsilon}^{n}}(x) d t=\int_{0}^{\infty} e^{-\lambda t} \mathbb{P}_{x}^{n}\left(\zeta^{\Omega_{\varepsilon}^{n}}>t\right) d t .
$$

Recall that (5.39) holds true. That is, from M-convergence of forms ([20]), we have that (Theorem 9)

$$
R_{\lambda}^{n} \mathbf{1}_{\Omega_{\varepsilon}^{n}}(x) \rightarrow \int_{0}^{\infty} e^{-\lambda t} \mathbb{P}_{x}\left(T_{c_{\infty}}>t\right) d t=R_{\lambda} \mathbf{1}_{\Omega}(x)
$$

strongly in $L^{2}(\Omega)$. The limit stopping time $T_{c_{\infty}}$ depends on $c_{\infty}$ and corresponds to the lifetime of the limit process. By construction we have that a.s. $\widehat{\zeta^{\infty}}=T_{c_{\infty}}$ and

$$
\lim _{n \rightarrow \infty} R_{\lambda}^{n} \mathbf{1}_{\Omega_{\varepsilon}^{n}}(x)=\lim _{n \rightarrow \infty} \widehat{R_{\lambda}^{n}} \mathbf{1}_{\Omega_{\varepsilon}^{n}}(x), \quad \forall x \in \Omega_{\varepsilon}^{1} .
$$

Now we show convergence in law.

Step 1) Let us consider a reflecting BM on $\Omega^{n}$, say $X^{n}$, and the first hitting time $\tau_{\Omega^{n}}$ on the pre-fractal boundary $\partial \Omega^{n}$. Let $X$ be a reflecting BM on $\Omega$ with $\Omega^{n} \uparrow \Omega$. We have that $X^{n} \stackrel{\text { law }}{\rightarrow} X$ as $n \rightarrow \infty$ ([22, Theorem 4.4 and Remark 2], [14, Section 2]). Consider now the killed process $X_{t}^{n}, t<\tau_{\Omega^{n}}$ started at $x \in \Omega_{\varepsilon}^{1}$. Here we can follow the same arguments as in [10, Theorem 4.1 and Theorem 3.3 ]. In particular, we consider the additive functional $A_{t}^{n}$ associated with $\tau_{\Omega^{n}}$ with Revuz measure $\mu_{A^{n}}=\infty_{D_{n}}$ where $D_{n}$ is the complement of $\Omega^{n}$. From stable convergence of multiplicative functionals we arrive at weak convergence of stopping times, that is $\tau_{\Omega^{n}} \stackrel{\text { law }}{\rightarrow} \tau_{\Omega}$. Therefore, we get a direct consequence of M-convergence of the associated form and tightness of $\mathbb{P}_{x}^{n}\left(\tau_{\Omega^{n}}>t\right.$ ) (indeed $\mathbb{E}_{x}^{n} \tau_{\Omega^{n}}$ is bounded by $\mathbb{E}_{x}^{n} \tau_{\Omega_{\varepsilon}^{1}}$ uniformly on $n$ ). Notice also that we arrive at the same result by considering Dirichlet 
condition on $\partial \Omega^{n}$ and the corresponding (killed) process on $\Omega^{n}$. The variational approach has been treated in [17].

Step 2) Now we focus on the family $\left\{\mathbb{P}_{x}^{n} ; x \in \Omega_{\varepsilon}^{n}\right\}$. Let us write $X_{t}^{x, n}=\left\{B_{t}^{\nu(n), *}\right.$ started from $\left.x \in \Omega_{\varepsilon}^{n}=\overline{\Omega^{n}} \cup \Sigma^{n}\right\} m$-a.e.. We first observe that

$$
\forall n, \quad X_{t}^{x, n} \in \partial \quad \text { if and only if } \quad\left(t>\zeta^{\Omega_{\varepsilon}^{n}}\right) \vee\left(X_{0}^{x, n} \in \partial\right) .
$$

Since $x \in \overline{\Omega_{\varepsilon}^{1}} \backslash \Omega_{\varepsilon}^{n} \Rightarrow X_{0}^{x, n} \in \partial$, for $n \rightarrow \infty$ we can consider starting points $x \in \Omega_{\varepsilon}^{1}$. Notice also that, in the Neumann case, the cemetery point is assumed to be $\partial=\{\emptyset\}$ and such that $m(\partial)=0$. This corresponds to $\operatorname{Cap}_{1}\left(\overline{\Omega_{\varepsilon}^{1}} \backslash \Omega^{n}\right) \rightarrow 0$. Thus, for $x \in \Omega_{\varepsilon}^{1}$ we write

$$
\begin{aligned}
& \mathbb{P}_{x}^{n}\left(\widehat{\zeta^{\Omega^{n}}}>t\right) \\
= & \left.\mathbb{P}_{x}^{n} \widehat{\left(\zeta^{\Omega^{n}}\right.}>t,\left(t<\tau_{\Omega^{n}}\right) \cup\left(t \geq \tau_{\Omega^{n}}\right)\right) \\
= & \mathbb{E}_{x}^{n}\left[e^{-c_{n} L_{t}^{\partial \Omega^{n}}} \mid t<\tau_{\Omega^{n}}\right] \mathbb{P}_{x}^{n}\left(t<\tau_{\Omega^{n}}\right)+\mathbb{E}_{x}^{n}\left[e^{-c_{n} L_{t}^{\partial \Omega^{n}}} \mid t \geq \tau_{\Omega^{n}}\right] \mathbb{P}_{x}^{n}\left(t \geq \tau_{\Omega^{n}}\right) \\
= & \mathbb{P}_{x}^{n}\left(\tau_{\Omega^{n}}>t\right)+\mathbb{E}_{x}^{n}\left[e^{-c_{n} L_{t}^{\partial \Omega^{n}}} \mid L_{t}^{\partial \Omega^{n}}>0\right] \mathbb{P}_{x}^{n}\left(t \geq \tau_{\Omega^{n}}\right) .
\end{aligned}
$$

From Proposition 5 and the fact that $\tau_{\Omega^{n}} \stackrel{\text { law }}{\rightarrow} \tau_{\Omega}$, we have that

$$
\mathbb{P}_{x}^{n}\left(\widehat{\zeta^{\Omega^{n}}}>t\right) \stackrel{w^{\star}}{\rightarrow} \mathbb{P}_{x}\left(\tau_{\Omega}>t\right)+\mathbb{E}_{x}\left[e^{-c_{\infty} L_{t}^{\partial \Omega}} \mid L_{t}^{\partial \Omega}>0\right] \mathbb{P}_{x}\left(t \geq \tau_{\Omega}\right)
$$

and therefore we obtain:

i) if $c_{n} \rightarrow c_{0}$, by considering that

$$
\begin{aligned}
& \mathbb{E}_{x}\left[e^{-c_{0} L_{t}^{\partial \Omega}}\right] \\
= & \mathbb{E}_{x}\left[e^{-c_{0} L_{t}^{\partial \Omega}} \mid L_{t}^{\partial \Omega^{n}}=0\right] \mathbb{P}_{x}^{n}\left(L_{t}^{\partial \Omega^{n}}=0\right)+\mathbb{E}_{x}\left[e^{-c_{0} L_{t}^{\partial \Omega}} \mid L_{t}^{\partial \Omega^{n}}>0\right] \mathbb{P}_{x}^{n}\left(L_{t}^{\partial \Omega^{n}}>0\right)
\end{aligned}
$$

and the fact that $\mathbb{P}_{x}\left(L_{t}^{\partial \Omega}=0\right)=\mathbb{P}_{x}\left(\tau_{\Omega}>t\right)$, we have

$$
\begin{aligned}
& \mathbb{E}_{x}\left[e^{-c_{0} L_{t}^{\partial \Omega}}\right] \\
= & \mathbb{E}_{x}\left[e^{-c_{0} L_{t}^{\partial \Omega}} \mid L_{t}^{\partial \Omega}=0\right] \mathbb{P}_{x}\left(\tau_{\Omega}>t\right)+\mathbb{E}_{x}\left[e^{-c_{0} L_{t}^{\partial \Omega}} \mid L_{t}^{\partial \Omega}>0\right] \mathbb{P}_{x}\left(t \geq \tau_{\Omega}\right) \\
= & \mathbb{P}_{x}\left(\tau_{\Omega}>t\right)+\mathbb{E}_{x}\left[e^{-c_{0} L_{t}^{\partial \Omega}} \mid L_{t}^{\partial \Omega}>0\right] \mathbb{P}_{x}\left(t \geq \tau_{\Omega}\right) .
\end{aligned}
$$

From Eq. 6.20, we obtain

$$
\mathbb{P}_{x}^{n}\left(\widehat{\zeta^{\Omega^{n}}}>t\right) \stackrel{w^{\star}}{\rightarrow} \mathbb{E}_{x}\left[e^{-c_{0} L_{t}^{\partial \Omega}}\right]=\mathbb{P}_{x}\left(\zeta^{\Omega}>t\right), \quad \forall t \geq 0,(x \in \bar{\Omega})
$$

and thus, a.s. $T_{c_{0}}=\zeta^{\Omega}$;

ii) if $c_{n} \rightarrow 0, \mathbb{P}_{x}^{n}\left(\widehat{\zeta^{\Omega^{n}}}>t\right) \stackrel{w^{\star}}{\rightarrow} \mathbb{P}_{x}\left(\tau_{\Omega}>t\right)+\mathbb{P}_{x}\left(t \geq \tau_{\Omega}\right)=1$ for all $t \geq 0, x \in \bar{\Omega}$ and

$$
\mathbb{P}_{x}\left(\omega: \lim _{n \rightarrow \infty} \widehat{\zeta^{\Omega^{n}}}(\omega)=\infty\right)=1=\mathbb{P}_{x}\left(\omega: T_{0}(\omega)=\infty\right)
$$

iii) if $c_{n} \rightarrow \infty, \mathbb{P}_{x}^{n}\left(\widehat{\zeta^{\Omega^{n}}}>t\right) \stackrel{w^{\star}}{\rightarrow} \mathbb{P}_{x}\left(\tau_{\Omega}>t\right)$ for all $t>0,(x \in \Omega)$ and we conclude that a.s. $T_{\infty}=\tau_{\Omega}$.

Thus, we obtain that $\widehat{\zeta^{\Omega^{n}}} \stackrel{\text { law }}{\rightarrow} T_{c_{\infty}}$. 
With this at hand, we now continue the proof. Since $\widehat{\zeta^{\Omega^{n}}} \leq \zeta^{\Omega_{\varepsilon}^{n}}$ with probability one, we have that $\mathbb{P}_{x}^{n}\left(\zeta^{\Omega_{\varepsilon}^{n}}>t\right) \geq \mathbb{P}_{x}^{n}\left(\widehat{\zeta^{n}}>t\right)$. Thus, we have that $\widehat{\mathbf{P}_{t}^{n}} \mathbf{1} \leq \mathbf{P}_{t}^{n} \mathbf{1}$ and $\widehat{R_{\lambda}^{n}} \mathbf{1} \leq R_{\lambda}^{n} \mathbf{1}$ $m$-a.e. $x$. From the contraction property of $\mathbf{P}_{t}^{n}$ we have that

$$
\left\|\widehat{R_{\lambda}^{n}} f\right\|_{L^{2}\left(\Omega_{\varepsilon}^{n}\right)} \leq\left\|R_{\lambda}^{n} f\right\|_{L^{2}\left(\Omega_{\varepsilon}^{n}\right)} \leq \lambda^{-1 / 2}\|f\|_{L^{2}\left(\Omega_{\varepsilon}^{n}\right)}
$$

for all measurable functions $f$. Strong convergence of resolvents in $L^{2}\left(\Omega^{*}\right)$ implies that, for $f \in L^{2}\left(\Omega_{\varepsilon}^{n}\right)$,

$$
\lim _{n \rightarrow \infty}\left\|R_{\lambda}^{n} f\right\|_{L^{2}\left(\Omega_{\varepsilon}^{n}\right)}=\left\|R_{\lambda} f\right\|_{L^{2}(\Omega)} .
$$

Since $R_{\lambda}^{n} f$ is uniformly bounded, $\left\|\widehat{R_{\lambda}^{n}} f\right\|_{L^{2}\left(\Omega_{\varepsilon}^{n}\right)}<C$ for all $n$. From this and convergence a.e. we conclude that $\widehat{R_{\lambda}^{n}} f \rightarrow R_{\lambda} f$ weakly in $L^{2}(\Omega)$. Convergence of $\widehat{R_{\lambda}^{n}} f$ implies that

$$
\liminf _{n \rightarrow \infty}\left\|\widehat{R_{\lambda}^{n}} f\right\|_{L^{2}\left(\Omega_{\varepsilon}^{n}\right)} \geq\left\|R_{\lambda} f\right\|_{L^{2}(\Omega)} .
$$

Since we have that

$$
\begin{aligned}
\left\|R_{\lambda} f\right\|_{L^{2}(\Omega)} & \leq \liminf _{n \rightarrow \infty}\left\|\widehat{R_{\lambda}^{n}} f\right\|_{L^{2}\left(\Omega_{\varepsilon}^{n}\right)} \\
& \leq \liminf _{n \rightarrow \infty}\left\|R_{\lambda}^{n} f\right\|_{L^{2}\left(\Omega_{\varepsilon}^{n}\right)}=\lim _{n \rightarrow \infty}\left\|R_{\lambda}^{n} f\right\|_{L^{2}\left(\Omega_{\varepsilon}^{n}\right)}=\left\|R_{\lambda} f\right\|_{L^{2}(\Omega)}
\end{aligned}
$$

we conclude that

$$
\lim _{n \rightarrow \infty}\left\|R_{\lambda}^{n} f\right\|_{L^{2}\left(\Omega_{\varepsilon}^{n}\right)}=\lim _{n \rightarrow \infty}\left\|\widehat{R_{\lambda}^{n}} f\right\|_{L^{2}\left(\Omega_{\varepsilon}^{n}\right)} .
$$

Weak convergence of $\widehat{R_{\lambda}^{n}} f$ together with Eq. 6.22 says that $\widehat{R_{\lambda}^{n}} f \rightarrow R_{\lambda} f$ strongly in $L^{2}(\Omega)$.

Now we consider $f_{n}$ as in Eq. 2.26. Since, from Eq. 6.21,

$$
\begin{aligned}
\left\|\widehat{R_{\lambda}^{n}} f_{n}-R_{\lambda} f\right\|_{L^{2}(\Omega)} & \leq\left\|\widehat{R_{\lambda}^{n}} f_{n}-\widehat{R_{\lambda}^{n}} f\right\|_{L^{2}(\Omega)}+\left\|\widehat{R_{\lambda}^{n}} f-R_{\lambda} f\right\|_{L^{2}(\Omega)} \\
& \leq \lambda^{-1 / 2}\left\|f_{n}-f\right\|_{L^{2}(\Omega)}+\left\|\widehat{R_{\lambda}^{n}} f-R_{\lambda} f\right\|_{L^{2}(\Omega)}
\end{aligned}
$$

we get the strong convergence of $\widehat{R_{\lambda}^{n}} f_{n}\left(\right.$ or $\left.\lambda \widehat{R_{\lambda}^{n}} f_{n}\right)$.

In light of the previous results, we can study the convergence of Eq. 5.29 by considering the semigroup (6.17) and therefore the corresponding form. By taking into account (3.9) and (3.4), we can study the form

$$
\mathcal{E}_{0}^{\mu \widehat{A^{n}}}(u, v)=\tilde{a_{n}}(u, v)+\langle u, v\rangle_{\mu_{\widehat{A^{n}}}}, \quad u, v \in H^{1}\left(\mathbb{R}^{2}\right) \cap L^{2}\left(\mu_{\widehat{A^{n}}}\right)
$$

associated with

$$
\mathbb{E}_{x}^{n}\left[f_{n}\left(\widetilde{B_{t}^{\nu(n), *}}\right) e^{-c_{n} \int_{0}^{t} \mathbf{1}_{\partial \Omega^{n}\left(B_{s}^{\nu(n), *}\right)}} d s\right]
$$

where $\tilde{a_{n}}$ is defined in Eq. 5.32 and $\mu_{\widehat{A^{n}}}$ is supported on $\partial \Omega^{n}$.

Proof of Theorem 10 From Theorem 11 we have that

$$
\mathbb{E}_{x}^{n}\left[\int_{0}^{\tau_{\Omega_{\varepsilon}^{n}}} e^{-\lambda t-\delta_{n} t} f_{n}\left(\widetilde{B_{t}^{\nu, *}}\right) \widehat{M_{t}^{n}} d t\right] \rightarrow R_{\lambda} f(x)=\mathbb{E}_{x}\left[\int_{0}^{\infty} e^{-\lambda t-\delta_{0} t} f\left(B_{t}^{+}\right) M_{t} d t\right]
$$

strongly in $L^{2}(\Omega)$ where the multiplicative functional $\widehat{M_{t}^{n}}$ and the killing functional are

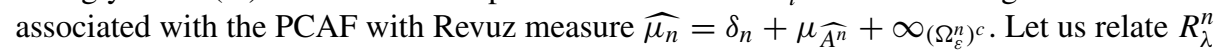
to $\overline{\mu_{n}}=\delta_{n}+\mu_{A^{n}}$ in the same sense. From Eq. 6.25 we have that, $\forall f \in C_{0}^{+}$,

$$
\int f d \overline{\mu_{n}} \rightarrow \int f d \varrho \Leftrightarrow \int f d \widehat{\mu_{n}} \rightarrow \int f d \varrho .
$$


Indeed, the resolvents identify uniquely the multiplicative functionals. Thus we get that

$$
\overline{\mu_{n}} \stackrel{v}{\rightarrow} \widehat{\mu_{\infty}} \text {. }
$$

This means equivalence between the corresponding additive functionals. Proposition 2 and Proposition 3 authorize us to study the asymptotic behaviour of

$$
\widehat{A_{t}^{n}}=\frac{c_{n}}{2}\left(A_{t}^{n+}+A_{t}^{n-}\right)
$$

and, by considering $\mu_{n}^{+}$and $\mu_{n}^{-}$, with Eqs. 3.5 and 3.4 in mind we get that

$$
\lim _{\lambda \rightarrow \infty}\left\langle\lambda U_{\lambda}^{n} f, m_{\varepsilon}^{n}\right\rangle=c_{n} \sigma_{n} \int_{\partial \Omega^{n}} f(x) \mathbf{1}_{\partial \Omega^{n}}(x) d \mathfrak{s}=\left\langle f, \mu_{\widehat{A^{n}}}\right\rangle .
$$

From Eq. 6.26, we get that

$$
\overline{\mu_{\infty}}=\delta_{0}+\left\{\begin{array}{lll}
c_{0} \mu_{\alpha} \mathbf{1}_{\partial \Omega}+\infty \bar{\Omega}^{c}, & c_{\infty}=c_{0} \in(0, \infty) & \text { (elastic kill), } \\
0+\infty \bar{\Omega}^{c}, & c_{\infty}=0 & \text { (no kill), } \\
\infty_{\Omega^{c}}, & c_{\infty}=\infty & \text { (kill on the boundary). }
\end{array}\right.
$$

With Eqs. 5.9 and 5.8 in mind, we write

$$
\lim _{n \rightarrow \infty} c_{n}=\lim _{n \rightarrow \infty} \frac{\sigma_{n}}{\operatorname{tick}\left(\Sigma^{n}\right)} \int_{\partial \Omega^{n}} \mathbb{P}_{x}^{n}\left(B_{t}^{v(n), *} \in \Sigma^{n}\right) m_{\varepsilon}^{n}(d x)
$$

where $w^{n} / \operatorname{tick}\left(\Sigma^{n}\right) \rightarrow 1$ as $n \rightarrow \infty\left(\operatorname{tick}\left(\Sigma^{n}\right)\right.$ is the thickness of the fiber).

iii) If $c_{n} \rightarrow \infty$, then point iii) of the proof of Theorem 11 holds and is also in accord with Eq. 6.27, $\tau_{\Omega_{\varepsilon}^{n}} \rightarrow \tau_{\Omega}$ faster than $B_{t}^{\nu(n), *} \rightarrow B_{t}^{+}$. The BM is killed on the boundary. On the other hand, if $\tau_{\Omega_{\varepsilon}^{n}} \rightarrow \tau_{\Omega}$, the lifetime on $\Omega$ of the limit process is exactly $\tau_{\Omega}$. Then for all $x \in \Omega$ we have that $\mathbb{P}_{x}\left(L_{t}^{\partial \Omega}=0\right)=1$ for all $t<\tau_{\Omega}$ which means that

$$
\forall x \in \Omega, \quad \mathbb{P}_{x}\left(e^{-c_{n} L_{t}^{\partial \Omega}}=1, t<\tau_{\Omega}\right)=1, \quad \text { for all } n \text { (for all } c_{n} \geq 0 \text { ). }
$$

This justifies the convention $\infty \cdot 0=0$. Moreover, if the lifetime is $\tau_{\Omega}$, from Eq. 6.3, it must be that $\zeta^{n} \rightarrow 0$, that is $c_{n} \rightarrow \infty$. Thus, $\tau_{\Omega_{\varepsilon}^{n}} \rightarrow \tau_{\Omega}$ if and only if condition (2.31) holds and $\partial \Omega$ becomes a Dirichlet boundary as $n \rightarrow \infty$. We get that $\tau_{\Omega_{\varepsilon}^{n}} \rightarrow \tau_{\Omega} \Leftrightarrow$ $c_{n} \rightarrow \infty$.

ii) If $c_{n} \rightarrow 0$, then according with Eq. 6.27 we say that $B_{t}^{\nu(n), *} \rightarrow B_{t}^{+}$on $\Omega$ for $t \geq 0$ and therefore, $\zeta^{\Omega^{n}} \rightarrow \infty$. On the other hand, Remark 3and Remark 4 say that $c_{n} \rightarrow$ $0 \Rightarrow \widehat{\zeta^{\Omega^{n}}} \rightarrow \infty$. Since $\widehat{\zeta^{\Omega^{n}}} \leq \zeta^{\Omega_{\varepsilon}^{n}}, \widehat{\zeta^{\Omega^{n}}} \rightarrow \infty$ as well as the lifetime $\zeta^{\Omega_{\varepsilon}^{n}} \rightarrow \infty$. Moreover, $\zeta^{\Omega_{\varepsilon}^{n}} \rightarrow \infty \Rightarrow c_{n} \rightarrow 0$ by using Remark 3 .

i) Since $i$ i) and iii) hold true, if $c_{n} \rightarrow c_{0}$ and $c_{0} \neq 0$ or $c_{0} \neq \infty$, then $\zeta^{\Omega_{\varepsilon}^{n}} \rightarrow T$ and $T \neq \infty$ or $T \neq \tau_{\Omega}$. In particular, $T \in\left(\tau_{\Omega}, \infty\right)$ is a random variable depending on $c_{0} \in(0, \infty)$. From Theorem 11 we have that $T=\zeta^{\Omega}$ is an exponential random variable with parameter $c_{0}$. Thus, $c_{n} \rightarrow c_{0} \Leftrightarrow \zeta^{\Omega_{\varepsilon}^{n}} \rightarrow \zeta^{\Omega}$. In this case (and also the previous as particular cases) we recover the results about the asymptotic of Robin problems on pre-fractal domains ([17]) by considering the forms (6.23).

Let us focus on Remark 1. We observe that, as $n \rightarrow \infty$,

$$
c_{n} \rightarrow c_{0}>0 \quad \Leftrightarrow \quad \operatorname{Cap}_{1}\left(\mathbb{R}^{2} \backslash \Omega\right)>0 \quad \text { (transient case) }
$$

where $\Rightarrow$ immediately follows and $\Leftarrow$ is obtained from $i i i)$ and $i$ ). Moreover, from $i i$ ),

$$
c_{n} \rightarrow 0 \quad \Leftrightarrow \quad \operatorname{Cap}_{1}\left(\mathbb{R}^{2} \backslash \Omega\right)=0 \quad \text { (recurrent case). }
$$


Indeed, the process $X_{t}$ is transient iff $\operatorname{Cap}_{1}\left(\mathbb{R}^{2} \backslash \Omega\right)>0$ ([24, Proposition 3.5.10]). A vanishing capacity can be related with the hitting distribution and in particular with the recurrence of the corresponding process. Let us consider the hitting distribution $H_{\Lambda^{c}}^{\alpha} \mathbf{1}_{E}(x)=\mathbb{E}_{x}\left[e^{-\alpha \tau_{\Lambda}} \mathbf{1}_{E}\left(B_{\tau_{\Lambda}}\right)\right]$ where the expected value is taken under (4.3). For $\alpha>0$, $H_{\Lambda^{c}}^{\alpha} \mathbf{1}(x)=\mathbb{E}_{x}\left[e^{-\alpha \tau_{\Lambda}}\right]$. Since $\mathbb{R}^{2} \backslash \Omega^{n}$ is a decreasing sequence of sets of finite capacity, from [30, Theorem 4.2.1] we have that

$$
\mathrm{Cap}_{1}\left(\mathbb{R}^{2} \backslash \Omega^{n}\right) \rightarrow 0 \quad \text { iff } \quad H_{\mathbb{R}^{2} \backslash \Omega^{n}}^{1} \mathbf{1}(x) \rightarrow 0 \text { q.e. }
$$

and thus, for $x \in \Omega_{\varepsilon}^{1}, \mathbb{P}_{x}\left(\tau_{\Omega^{n}}<\infty\right) \rightarrow 0$ (see also Eq. 6.2).

Acknowledgments We are very grateful to the reviewer for the precious and accurate reading of the previous versions of the work.

\section{References}

1. Acerbi, E., Buttazzo, G.: Reinforcement problems in the calculus of variations. Ann. Inst. H. Poincaré Anal. Non Lin. 3(4), 273-284 (1986)

2. Aldous, D.: Stopping times and tightness. Ann. Probab. 6(2), 335-340 (1978)

3. Aldous, D.: Stopping times and tightness. II. Ann. Probab. 17(2), 586-595 (1989)

4. Appuhamillage, T., Bokil, V., Thomann, E., Waymire, E., Wood, B.: Occupation and local times for skew Brownian motion with applications to dispersion across an interface. Ann. Appl. Probab. 21(1), 183-214 (2011)

5. Atar, R., Budhiraja, A.: On the multi-dimensional skew Brownian motion. Stoch. Proc. Appl. 125, 19111925 (2015)

6. Bass, R.F., Burdzy, K.: Pathwise uniqueness for reflecting Brownian motion in certain planar Lipschitz domains. Electron. Comm. Probab. 11, 178-181 (2006)

7. Bass, R.F., Burdzy, K., Chen, Z.: Uniqueness for reflecting Brownian motion in lip domains. Ann. Inst. H. Poincaré Probab. Stat. 41(2), 197-235 (2005)

8. Bass, R.F., Burdzy, K., Chen, Z.: On the Robin problem in fractal domains. Proc. Lond. Math. Soc. (3) 96(2), 273-311 (2008)

9. Bass, R.F., Hsu, P.: Some potential theory for reflecting Brownian motion in holder and Lipschitz domains. Ann. Probab. 19(2), 486-508 (1991)

10. Baxter, J., Dal Maso, G., Mosco, U.: Stopping times and $\Gamma$-convergence. Trans. Amer. Math. Soc. 303(1), 1-38 (1987)

11. Blumenthal, R.M., Getoor, R.K.: Additive functionals of Markov processes in duality. Trans. Amer. Math. Soc. 112, 131-163 (1964)

12. Blumenthal, R.M., Getoor, R.K.: Markov Processes and Potential Theory. Academic Press, New York (1968)

13. Brezis, H., Caffarelli, L.A., Friedman, A.: Reinforcement problems for elliptic equations and variational inequalities. A.n. Mat. Pura Appl. (4) 123, 219-246 (1980)

14. Burdzy, K., Chen, Z.-Q.: Weak convergence of reflecting Brownian motions. Elect. Comm. Probab. 3, 29-33 (1998)

15. Burdzy, K., Chen, Z.-Q., Marshall, D.: Traps for reflected Brownian motion. Math. Z 252(1), 103-132 (2006)

16. Buttazzo, G., Dal Maso, G., Mosco, U.: Asymptotic Behaviour for Dirichlet Problems in Domains Bounded by Thin Layers, Partial Differential Equations and the Calculus of Variations, Vol. I, Progr. Nonlinear Differential Equations Appl., vol. 1, pp. 193-249. Birkhäuser, Boston (1989)

17. Capitanelli, R.: Asymptotics for mixed Dirichlet-Robin problems in irregular domains. J. Math. Anal. Appl. 362(2), 450-459 (2010)

18. Capitanelli, R., Lancia, M.R., Vivaldi, M.A.: Insulating layers of fractal type. Diff. Integr. Equ. 26(9-10), 1055-1076 (2013)

19. Capitanelli, R., Vivaldi, M.A.: Insulating layers on fractals. J. Diff. Equ. 251(4-5), 1332-1353 (2011)

20. Capitanelli, R., Vivaldi, M.A.: On the Laplacean transfer across fractal mixtures. Asymp. Anal. 83(1-2), 1-33 (2013) 
21. Chan, T.: Occupation times of compact sets by planar Brownian motion. Ann. Inst. H. Poincaré Probab. Stat. 30(2), 317-329 (1994)

22. Chen, Z.-Q.: On reflecting diffusion processes and Skorokhod decompositions. Probab. Theory Related Fields 94(3), 281-315 (1993)

23. Chen, Z.-Q., Fukushima, M., Ying, J.: Traces of symmetric Markov processes and their characterizations. Ann. Probab. 34(3), 1052-1102 (2006)

24. Chen, Z.-Q., Fukushima, M.: Symmetric Markov Processes, Time Change, and Boundary Theory, London Mathematical Society Monographs. Princeton University Press (2012)

25. Chung, K.L.: Probabilistic approach in potential theory to the equilibrium problem. Ann. Inst. Fourier (Grenoble) 23(3), 313-322 (1973)

26. Dembo, A., Peres, Y., Rosen, J., Zeitouni, O.: Thick points for planar Brownian motion and the ErdösTaylor conjecture on random walk. Acta Math. 186(2), 239-270 (2001)

27. Falconer, K.J.: The Geometry of Fractal Sets. Cambridge Tracts in Mathematics, vol. 85. Cambridge University Press, Cambridge (1986)

28. Fitzsimmons, P.J., Pitman, J.: Kac's moment formula and the Feynman-Kac formula for additive functionals of a Markov process. Stoch. Process Appl. 79(1), 117-134 (1999)

29. Fukushima, M.: A construction of reflecting barrier Brownian motions for bounded domains. Osaka J. Math. 4, 183-215 (1967)

30. Fukushima, M., Oshima, Y., Takeda, M.: Dirichlet Forms and Symmetric Markov Processes. Walter de Gruyter \& Co, New York (1994)

31. Getoor, R.K., Sharpe, M.J.: Last exit times and additive functionals. Ann. Probab. 1, 550-569 (1973)

32. Gyrya, P., Saloff-Coste, L.: Neumann and Dirichlet heat kernels in inner uniform domains, Société Mathématique de France, Astérisque No. 336 (2011)

33. Harrison, J.M., Shepp, L.A.: On skew Brownian motion. Ann. Probab. 9(2), 309-313 (1981)

34. Itô, K., McKean, H.P.: Diffusion and Their Sample Paths, 2nd eds. Springer-Verlag (1974)

35. Jonsson, A., Wallin, H.: Function spaces on subsets of $\mathbb{R}^{n}$. Math. Rep. 2(1), xiv+221 (1984)

36. Kallenberg, O.: Foundations of Modern Probability. Springer, New York (1997)

37. Karlin, S., Taylor, H.M.: A Second Course in Stochastic Processes. Academic Press, New York (1975)

38. Lang, R.: Effective conductivity and skew Brownian motion. J. Stat. Phys. 80(1-2), 125-146 (1995)

39. Lejay, A.: On the constructions of the skew Brownian motion. Probab. Surv. 3, 413-466 (2006)

40. Lejay, A., M.rtinez, M.: A scheme for simulating one-dimensional diffusion processes with discontinuous coefficients. Ann. Appl. Probab. 16(1), 107-139 (2006)

41. Mosco, U.: Convergence of convex sets and of solutions of variational inequalities. Adv. Math. 3, 510585 (1969)

42. Mosco, U.: Composite media and asymptotic Dirichlet forms. J. Funct. Anal. 123(2), 368-421 (1994)

43. Ouknine, Y.: Le "Skew-Brownian motion" et les processus qui en dérivent. Teor. Veroyatnost. i Primenen. 35(1), 173-179 (1990)

44. Portenko, N.I.: Diffusion processes with a generalized drift coefficient. Teor. Veroyatnost. i Primenen. 24(1), 62-77 (1979)

45. Portenko, N.I.: Stochastic differential equations with a generalized drift vector. Teor. Veroyatnost. i Primenen. 24(2), 332-347 (1979)

46. Ramirez, J.M.: Multi-skewed Brownian motion and diffusion in layered media. Proc. Amer. Math. Soc. 139(10), 3739-3752 (2011)

47. Revuz, D., Yor, M.: Continuous martingales and Brownian motion, 3rd edn. Springer-Verlag (1999)

48. Rogers, L.C.G., Williams, D.: Diffusions, Markov Processes and Martingales: Volume 1, Foundations. Cambridge University Press (2000)

49. Rogers, L.C.G., Williams, D.: Diffusions, Markov Processes and Martingales: Volume 2, Itô Calculus. Cambridge University Press (2000)

50. Stollmann, P.: A convergence theorem for Dirichlet forms with applications to boundary value problems with varying domains. Math. Z 219(2), 275-287 (1995)

51. Takemura, T.: Convergence of time changed skew product diffusion processes. Potential Anal. 38(1), 31-55 (2013)

52. Trutnau, G.: Skorokhod decomposition of reflected diffusions on bounded Lipschitz domains with singular non-reflection part. Probab. Theory Related Fields 127(4), 455-495 (2003)

53. Trutnau, G.: Multidimensional Skew Reflected Diffusions. Stochastic Analysis: Classical and Quantum, pp. 228-244. World Sci. Publ., Hackensack (2005)

54. Walsh, J.B.: A diffusion with a discontinuous local time. Société Mathématique de France Astérisque 52-53, 37-45 (1978)

55. Weinryb, S.: Homogénéisation pour des processus associés à des frontières perméables. Ann. Inst. H. Poincaré Probab. Stat. 20(4), 373-407 (1984) 